\title{
Distribution function approach to redshift space distortions. Part III: halos and galaxies
}

\author{
Okumura, Teppei ; Seljak, Uroš ; Desjacques, Vincent
}

\begin{abstract}
It was recently shown that the power spectrum in redshift space can be written as a sum of cross-power spectra between number weighted velocity moments, of which the lowest are density and momentum density. We investigate numerically the properties of these power spectra for simulated galaxies and dark matter halos and compare them to the dark matter power spectra, generalizing the concept of the bias in density-density power spectra. Because all of the quantities are number weighted this approach is well defined even for sparse systems such as massive halos. This contrasts to the previous approaches to RSD where velocity correlations have been explored, but velocity field is a poorly defined concept for sparse systems. We find that the number density weighting leads to a strong scale dependence of the bias terms for momentum density auto-correlation and cross-correlation with density. This trend becomes more significant for the more biased halos and leads to an enhancement of RSD power relative to the linear theory. Fingers-of-god effects, which in this formalism come from the correlations of the higher order moments beyond the momentum density, lead to smoothing of the power spectrum and can reduce this enhancement of power from the scale dependent bias, but are relatively small for halos with no small scale velocity dispersion. In comparison, for a more realistic galaxy sample with satellites the small scale velocity dispersion generated by satellite motions inside the halos leads to a larger power suppression on small scales, but this depends on the satellite fraction and on the details of how the satellites are distributed inside the halo. We investigate several statistics such as the two-dimensional power spectrum $\mathrm{P}(\mathrm{k}$,$) , where is the angle between the Fourier mode and line of sight, its multipole$ moments, its powers of 2 , and configuration space statistics. Overall we find that the nonlinear effects in realistic galaxy samples such as luminous red galaxies affect the redshift space clustering on very large scales: for example, the quadrupole moment is affected by $10 \%$ for $\mathrm{k}<0.1 \mathrm{hMpc}-1$, which means that these effects need to be understood if we want to extract cosmological information from the redshift space distortions.
\end{abstract}

DOI: https://doi.org/10.1088/1475-7516/2012/11/014

Posted at the Zurich Open Repository and Archive, University of Zurich

ZORA URL: https://doi.org/10.5167/uzh-70069

Journal Article

Originally published at:

Okumura, Teppei; Seljak, Uroš; Desjacques, Vincent (2012). Distribution function approach to redshift space distortions. Part III: halos and galaxies. Journal of Cosmology and Astroparticle Physics, 2012(11):14.

DOI: https://doi.org/10.1088/1475-7516/2012/11/014 


\title{
Distribution function approach to redshift space distortions. Part III: halos and galaxies
}

\section{Teppei Okumura, ${ }^{a}$ Uroš Seljak, $^{a, b, c}$ and Vincent Desjacques ${ }^{d}$}

\author{
${ }^{a}$ Institute for the Early Universe, Ewha Womans University, Seoul 120-750, S. Korea \\ ${ }^{b}$ Department of Physics, Department of Astronomy, and Lawrence Berkeley National Laboratory, \\ University of California, Berkeley, California 94720, USA \\ ${ }^{c}$ Institute of Theoretical Physics, University of Zurich, 8057 Zurich, Switzerland \\ ${ }^{d}$ Département de Physique Théorique and Center for Astroparticle Physics (CAP), Université de \\ Genève, 1211 Genève, Switzerland
}

E-mail: teppei@ewha.ac.kr, useljak@berkeley.edu,dvince@physik.uzh.ch

\begin{abstract}
It was recently shown that the power spectrum in redshift space can be written as a sum of cross-power spectra between number weighted velocity moments, of which the lowest are density and momentum density. We investigate numerically the properties of these power spectra for simulated galaxies and dark matter halos and compare them to the dark matter power spectra, generalizing the concept of the bias in density-density power spectra. Because all of the quantities are number weighted this approach is well defined even for sparse systems such as massive halos. This contrasts to the previous approaches to RSD where velocity correlations have been explored, but velocity field is a poorly defined concept for sparse systems. We find that the number density weighting leads to a strong scale dependence of the bias terms for momentum density auto-correlation and cross-correlation with density. This trend becomes more significant for the more biased halos and leads to an enhancement of RSD power relative to the linear theory. Fingers-of-god effects, which in this formalism come from the correlations of the higher order moments beyond the momentum density, lead to smoothing of the power spectrum and can reduce this enhancement of power from the scale dependent bias, but are relatively small for halos with no small scale velocity dispersion. In comparison, for a more realistic galaxy sample with satellites the small scale velocity dispersion generated by satellite motions inside the halos leads to a larger power suppression on small scales, but this depends on the satellite fraction and on the details of how the satellites are distributed inside the halo. We investigate several statistics such as the two-dimensional power spectrum $P(k, \mu)$, where $\mu$ is the angle between the Fourier mode and line of sight, its multipole moments, its powers of $\mu^{2}$, and configuration space statistics. Overall we find that the nonlinear effects in realistic galaxy samples such as luminous red galaxies affect the redshift space clustering on very large scales: for example, the quadrupole moment is affected by $10 \%$ for $k<0.1 h \mathrm{Mpc}^{-1}$, which means that these effects need to be understood if we want to extract cosmological information from the redshift space distortions.
\end{abstract}

Keywords: galaxy clustering, power spectrum, redshift surveys

ArXiv ePrint: 1206.XXXX 


\section{Contents}

1 Introduction $\quad 1$

2 Redshift-space distortions from the distribution function 3

2.1 Angular decomposition and relation to Legendre multipole moments 4

$3 \quad N$-body simulations $\quad \mathbf{5}$

$\begin{array}{lll}3.1 & \text { Dark matter halo and galaxy catalogs } & 5\end{array}$

$\begin{array}{llr}3.2 & \text { Power spectra and two-point correlation functions } & 6\end{array}$

4 Numerical analysis $\quad 7$

$\begin{array}{lll}4.1 & \text { Power spectra of dark matter halos and galaxies } & 7\end{array}$

4.2 Biasing of moments correlators $\quad 8$

$\begin{array}{llr}4.3 & \text { Relation to linear RSD } & 11\end{array}$

$\begin{array}{ll}4.4 \\ \text { Legendre moments of redshift-space power spectrum } & 13\end{array}$

5 Expansion in powers of $\mu^{2} \quad 15$

$\begin{array}{lll}5.1 \mu^{0} \text { term } & 15\end{array}$

$\begin{array}{ll}5.2 \mu^{2} \text { terms } & 15\end{array}$

$\begin{array}{ll}5.3 \mu^{4} \text { terms } & 17\end{array}$

$\begin{array}{lr}5.4 \mu^{6} \text { and } \mu^{8} \text { terms } & 19\end{array}$

5.5 Comparison of $\mu^{2}$ expansions with Legendre expansions 20

6 Configuration-space analysis $r$

7 Conclusions $r$

\section{Introduction}

Galaxy redshift surveys are one of the most powerful tools to probe cosmological models [1]. Galaxy distribution in redshift surveys is distorted through the Doppler shift by peculiar velocities of galaxies along the line of sight. Thus the measured redshift of the galaxy provide not only the information of the radial distance but also that of the radial velocity. This effect, so called redshift-space distortions (RSD), induces anisotropies in the galaxy clustering and allows one to measure the amplitude of density fluctuations times the rate of growth of structure on large scales, $f=d \ln D / d \ln a$, with $D$ the linear growth factor $[2,3]$. Cosmological models in different gravity theories can have a different value of $f$, thus RSD are a promising tool to investigate gravity theories [e.g., 4-7]. So far RSD have been analyzed in many galaxy surveys to determine the cosmological models [e.g., 6, 8-16]. However, it was shown by [17-21] that the parameter reconstructed from the redshift-space distortions can have scale dependent bias, which indicates a breakdown of the linear theory predictions. These effects show up on relatively large scales, suggesting one must go beyond the linear theory in the analysis of RSD.

Given the high precision of the future surveys, correspondingly more accurate theoretical predictions become essential for their interpretation. Recently there have been many studies to predict the power spectrum in nonlinear regime beyond the framework of the standard perturbation theory (SPT) [22-28]. Similarly, initial RSD work was based on the lowest order SPT [29-32]. However, as pointed out by [30, 33], SPT in redshift space breaks down at larger scales than in real space because of nonlinear redshift distortion effects. Sometimes this is attributed to the so-called Fingers-of-God (FoG) effect [34]. However, we will argue that a more important effect is the scale dependent biasing. Recent development using more sophisticated perturbation methods applicable to the redshift-space power spectrum includes [35-38]. 
There is another issue that needs to be taken into account to achieve accurate theoretical predictions of RSDs. Galaxies, or dark matter halos within which all galaxies are expected to form, are a biased tracer of dark matter, as their clustering strength is typically enhanced relative to the dark matter, which is known as biasing [39, 40]. Because of the existence of the bias, RSD on linear scales in galaxy surveys allow us to measure the linear RSD parameter $\beta=f / b$, where $b$ is the bias parameter. This can be combined with the auto-correlation of galaxies to eliminate the bias and measure $f A$, where $A$ is the amplitude of density fluctuations (often parameterized with $\sigma_{8}$ ). Recently it was shown by [19] using linear theory that the RSD parameters reconstructed from the clustering of halos have strong halo-mass and scale dependence even on large scales. It is, however, not trivial to incorporate the bias into an analytical framework of nonlinear perturbation theory, since the galaxy formation is a highly nonlinear process. One attempt was presented by [41, 42] in redshift space using the Lagrangian perturbation theory. However, [43] show that the formula of [41] seriously fails to predict the quadrupole and hexadecapole moments of the redshift-space correlation function even on very large scales. [44] extended the formalism of [36] by combining with a simple halo bias scheme and tested it using dark matter halo catalogs from $N$-body simulations (see also [45]). There are also several studies to attempt to eliminate such nuisance effects by combining RSD to the other measurement. [46] proposed a method to eliminate the uncertainty of the galaxy biasing by combining weak gravitational lensing, galaxy clustering and RSD (see [47] for the observational result). Similarly, [48] developed an approach for using galaxy-galaxy weak lensing to model the FoG effect in RSD measurements.

A recent paper [38] (Part I in the series of papers studying RSD) has developed a phase space distribution function approach to RSD where the redshift-space density can be written as a sum over mass or number weighted moments of radial velocity, which are integrals of powers of velocity over the momentum part of the phase space distribution function. The corresponding RSD power spectrum can be written as a sum over auto and cross-correlators of these moments. In [49] (Part II) we analyzed a large set of $N$-body simulations to test how accurately this formalism predicts the true power spectrum of dark matter as a function of terms included. The expansion was compared to the Legendre moments, the monopole, quadrupole and hexadecapole moments. These comparisons revealed that the expansion is accurate within a few percent up to $k \simeq 0.15(1+z) h \mathrm{Mpc}^{-1}$ if the corrections up to the 6 th order are taken into account. We also presented a resummation of some of the terms into a power suppression factors called the FoG kernel. This FoG model has validity comparable or better than the 6th order summations and predicts the monopole power spectrum with a few percent accuracy up to $k \simeq 0.4 h \mathrm{Mpc}^{-1}$ at $z=0$ and for $k<1 h \mathrm{Mpc}^{-1}$ at $z=0.5$ and 1. [38] has also shown that the moments can be decomposed into helicity eigenstates, which are eigenmodes under rotation around direction of $\mathbf{k}$ vector. Only equal helicity eigenstates correlate, leading to a specific angular structure of the correlators. This analysis shows that if one expands the series into powers of $\mu^{2 j}$, a finite number of terms contribute at each (finite) order. This suggests that RSD can be better understood in terms of this expansion rather than the Legendre moments usually used. Using the angular decomposition the individual terms for the coefficients of $\mu^{2 j}$ for the dark matter power spectrum were determined. Detailed comparison of the numerical results to perturbation theory predictions will be made in [50] (Part IV).

This paper is Part III in this series. Because RSD is described by number-weighted velocity moment correlators, there is a particular advantage when we analyze galaxies and dark matter halos, since for sparse systems volume-weighted velocity moments cannot be easily defined. In this paper we test our formalism to describe the redshift-space power spectrum of galaxies and halos in nonlinear regime using a large set of cosmological $N$-body simulations, as well as present the individual terms of expansion for comparison against each other, as an extension of the analysis of the dark matter power spectrum in Part II [49]. The structure of this paper is as follows. In section 2 we briefly review the distribution function approach to RSD and extend it for biased objects such as halos. Section 3 describes the $N$-body simulations and how to measure the two-point statistics used in this paper. Section 4 presents the power spectra of the number-weighted velocity moments and their contributions to the full 2-d spectrum in redshift space. We also discuss in detail properties of biasing of halos and galaxies using these power spectra. In section 5 we apply our formalism to powers of $\mu$ 
expansion, showing individual contributions to $\mu^{0}, \mu^{2}, \cdots, \mu^{8}$ terms in $P^{s}$. In section 6 we present the redshift-space correlation functions of halos and galaxies and compare to the power spectrum analysis. Section 7 is devoted to conclusions of this paper.

\section{Redshift-space distortions from the distribution function}

Throughout this paper we adopt a phase-space distribution function approach to describe redshiftspace distortions, proposed by [38, 51]. This approach was tested to dark matter simulations by [49], and a similar discussion is applicable to dark matter halos and galaxies. The exact evolution of collisionless particles is described by the Vlasov equation [1]. We thus start from the distribution function of particles $f(\mathbf{x}, \mathbf{q}, t)$ at phase-space position $(\mathbf{x}, \mathbf{q})$ in order to derive the perturbative redshift-space distortions. Here $\mathbf{x}$ is the comoving position and $\mathbf{q}=\mathbf{p} / a$ is the comoving momentum ( $\mathbf{p}$ is the proper momentum). The density field in redshift space is related to moments of distribution function as

$$
\delta_{s}^{m}(\mathbf{k})=\sum_{L=0} \frac{1}{L !}\left(\frac{i k_{\|}}{\mathcal{H}}\right)^{L} T_{\|}^{L, m}(\mathbf{k}),
$$

where the superscript $m$ denotes quantities for dark matter and $\mathcal{H}=a H$ where $H$ is the Hubble parameter. $T_{\|}^{L, m}(\mathbf{k})$ is the Fourier transform of $T_{\|}^{L, m}(\mathbf{x})$, defined as

$$
T_{\|}^{L, m}(\mathbf{x})=\frac{m_{p}}{\bar{\rho}} \int d^{3} \mathbf{q} f(\mathbf{x}, \mathbf{q}) u_{\|}^{L}=\left\langle\left(1+\delta^{m}(\mathbf{x})\right) u_{\|}^{L}(\mathbf{x})\right\rangle_{\mathbf{x}},
$$

where $u_{\|}$is the radial peculiar velocity, ${ }^{1} m_{p} u_{\|}=q_{\|}=\mathbf{q} \cdot \hat{r}, m_{p}$ is the particle mass, $\hat{r}$ is the unit vector pointing along the observer's line of sight and $\bar{\rho}$ is the mean mass density. The power spectrum in redshift space is then given by [38, 49],

$$
P_{m m}^{s}(\mathbf{k})=\sum_{L=0}^{\infty} \sum_{L^{\prime}=0}^{\infty} \frac{(-1)^{L^{\prime}}}{L ! L^{\prime} !}\left(\frac{i k \mu}{\mathcal{H}}\right)^{L+L^{\prime}} P_{L L^{\prime}}^{m m}(\mathbf{k}),
$$

where $k_{\|} / k=\cos \theta=\mu$ and $P_{L L^{\prime}}^{m m}(\mathbf{k}) \delta\left(\mathbf{k}-\mathbf{k}^{\prime}\right)=\left\langle T_{\|}^{L, m}(\mathbf{k})\left(T_{\|}^{* L^{\prime}, m}\left(\mathbf{k}^{\prime}\right)\right)\right\rangle$.

The model of the power spectrum for mass presented above can be extended to biased objects such as dark matter halos and galaxies without any assumption. It is given by simply replacing the superscript and subscript $m$ in equation (2.3) by $h$, denoting quantities for halos, as

$$
\begin{aligned}
P_{h h}^{s}(\mathbf{k}) & =\sum_{L=0}^{\infty} \sum_{L^{\prime}=0}^{\infty} \frac{(-1)^{L^{\prime}}}{L ! L^{\prime} !}\left(\frac{i k \mu}{\mathcal{H}}\right)^{L+L^{\prime}} P_{L L^{\prime}}^{h h}(\mathbf{k}) \\
& =\sum_{L=0}^{\infty} \frac{1}{L !^{2}}\left(\frac{k \mu}{\mathcal{H}}\right)^{2 L} P_{L L}^{h h}(\mathbf{k})+2 \sum_{L=0}^{\infty} \sum_{L^{\prime}>L} \frac{(-1)^{L^{\prime}}}{L ! L^{\prime} !}\left(\frac{i k \mu}{\mathcal{H}}\right)^{L+L^{\prime}} P_{L L^{\prime}}^{h h}(\mathbf{k}) .
\end{aligned}
$$

We will sometimes omit the super/subscript $m$ and $h$ in the following when a given equation holds for both dark matter and halos.

It is useful to compare this to Kaiser's linear theory prediction $[2,33]$. If we approximate the expression with the lowest 3 terms $P_{00}, P_{01}$ and $P_{11}$ and assume standard linear theory, we obtain the linear Kaiser formula, as

$$
P_{h h, \text { Kaiser }}^{s}(\mathbf{k})=\left(b+f \mu^{2}\right)^{2} P_{00, \text { lin }}^{m m}(k) ; \text { linear, }
$$

\footnotetext{
${ }^{1}$ Unlike the definition here, in our previous paper [49] the velocity $u_{\|}$and the velocity moments $T_{\|}^{L}$ were defined in comoving coordinates, thus $H$ instead of $\mathcal{H}$ was used in the formalism. These two expressions are essentially the same. However, in [49] there was an obvious typo in the definition of $u_{\|}$: it should have been $a m_{p} u_{\|}=q_{\|}$.
} 
where $b$ is the bias parameter (see section 4.2 below) and $f=d \ln D / d \ln a$ with $D$ the growth factor. If the nonlinear corrections for these terms are taken into account,

$$
\begin{aligned}
P_{h h, \text { Kaiser }}^{s}(\mathbf{k}) & =P_{00}^{h h}(k)+2 f \mu^{2}\left(\frac{i k}{\mathcal{H} \mu f}\right) P_{01}^{h h}(k)+f^{2} \mu^{4}\left(\frac{k}{\mathcal{H} \mu f}\right)^{2} P_{11}^{h h}(k) ; \text { nonlinear } \\
& =b^{2} P_{00}^{m m}(k)+2 b f \mu^{2}\left(\frac{i k}{\mathcal{H} \mu f}\right) P_{01}^{m m}(k)+f^{2} \mu^{4}\left(\frac{k}{\mathcal{H} \mu f}\right)^{2} P_{11}^{m m}(k),
\end{aligned}
$$

i.e., it is given by the lowest 3 terms $P_{00}, P_{01}$ and $P_{11}$. Since these terms have nonlinear corrections, we call this approximation the nonlinear Kaiser order approximation. Replacing these lowest 3 moments with the standard linear theory we obtain the original linear Kaiser model of equation (2.5), and

$$
P_{00, \operatorname{lin}}^{m m}(\mathbf{k})=\left(\frac{i k}{\mathcal{H} \mu f}\right) P_{01, \operatorname{lin}}^{m m}(\mathbf{k})=\left(\frac{i k}{\mathcal{H} \mu f}\right)^{2} P_{11, \text { lin }}^{m m}(\mathbf{k}) .
$$

Here we want to view this series simply as a series in $k_{\|}$, investigating the convergence as more terms are added.

Note that the calculations never require anything but simple power spectra of number-weighted powers of velocity to be computed from the simulations. These number-weighted quantities are welldefined even for sparse biased systems such as halos or galaxies. The order of $k_{\|}=k \mu$ needed for convergence to a given level of accuracy will inevitably increase as one goes to increasingly small scales, with the whole expansion eventually breaking down once $k \mu \sigma / \mathcal{H}>1$, where $\sigma$ is a typical velocity of the system.

\subsection{Angular decomposition and relation to Legendre multipole moments}

By performing helicity decomposition [38] show that the power spectrum can be written as

$$
P_{L L^{\prime}}(\mathbf{k})=\sum_{(l=L, L-2, . .)} \sum_{\left(l^{\prime}=L^{\prime}, L^{\prime}-2, . . . ; l^{\prime} \geq l\right)} \sum_{n=0}^{l} P_{l, l^{\prime}}^{L, L^{\prime}, n}(k) P_{l}^{n}(\mu) P_{l^{\prime}}^{n}(\mu),
$$

where $P_{l}^{n}(\mu)$ are the associated Legendre polynomials, which determine the angular dependence of the spherical harmonics. There are 5 numbers that describe these objects: $L$ and $L^{\prime}$ describe the power of two velocity moments we are correlating, $l, l^{\prime}$ describe the rank of the object, for example $l=1$ is rank-1, which is a 3 -d vector, $l=2$ is a 3 -d tensor etc. Finally, $n$ is the helicity eigennumber, ${ }^{2}$ which ranges between 0 and $l\left(l \leq l^{\prime}\right)$. Only equal helicity components of expansion have a non-vanishing correlator. There is a close relation between the order of the moments and their angular dependence. The lowest contribution in powers of $\mu$ to $P^{s}(k)$ is $\mu^{L+L^{\prime}}$ if $L+L^{\prime}$ is even or $\mu^{L+L^{\prime}+1}$ if $L+L^{\prime}$ is odd, and the highest is $\mu^{2\left(L+L^{\prime}\right)}$. Thus for $P_{00}(\mathbf{k})$ the only angular term is isotropic term $\left(\mu^{0}\right)$, for $P_{01}(\mathbf{k})$ the only angular term is $\mu^{2}, P_{11}(\mathbf{k})$ and $P_{02}(\mathbf{k})$ contain both $\mu^{2}$ and $\mu^{4}$ etc. Note that only even powers of $\mu$ enter in the final expression, as required by the symmetry. We can thus write

$$
P^{s}(\mathbf{k})=\sum_{L=0}^{\infty} \frac{1}{L !^{2}}\left(\frac{k}{\mathcal{H}}\right)^{2 L} \sum_{j=2 L}^{4 L} P_{L L}^{(j)}(\mathbf{k}) \mu^{j}+2 \sum_{L=0}^{\infty} \sum_{L^{\prime}>L} \frac{(-1)^{L}}{L ! L^{\prime} !}\left(\frac{i k}{\mathcal{H}}\right)^{L+L^{\prime}} \sum_{j=\left(L+L^{\prime}\right) \operatorname{or}\left(L+L^{\prime}+1\right)}^{2\left(L+L^{\prime}\right)} P_{L L^{\prime}}^{(j)}(\mathbf{k}) \mu^{j}
$$

so that terms $P_{L L^{\prime}}^{(j)}$ are coefficients in expansion in powers of $\mu^{j}$ of contributions of $L, L^{\prime}$ terms to $P^{s}$. The $j$ index has to be even, so the lowest order is either $L+L^{\prime}$ or $L+L^{\prime}+1$, whichever is even. These terms can be uniquely extracted from simulations from angular dependence of $P_{L L^{\prime}}$ terms and so we will focus on them, although sometimes it is useful to decompose them into the individual helicity eigenstates instead. We can write the redshift-space power spectrum described in terms of $\mu^{2}$ moments (equation $(2.10)$ ) as

$$
P^{s}(\mathbf{k})=\sum_{j=0,2,4, \cdots} P_{\mu^{j}}(k) \mu^{j}
$$

\footnotetext{
${ }^{2} m$ is usually used in place of $n$, but here the superscript $m$ is used to describe quantities for dark matter.
} 
Note that only even powers of $\mu$ enter in the final expression, as required by the symmetry. There is a close relation between the order of the moments and their angular dependence. The lowest contribution in powers of $\mu$ to $P^{s}(k)$ is $P_{00}(\mathbf{k})$, which is the only angular term is isotropic term $\left(\mu^{0}\right)$, for $P_{01}(\mathbf{k})$ the only angular term is $\mu^{2}, P_{11}(\mathbf{k})$ and $P_{02}(\mathbf{k})$ contain both $\mu^{2}$ and $\mu^{4}$ etc. There is always a finite number of terms contributing to a given order of $j$ : for $j=4$ we have 7 terms contributing to it.

The Legendre multipole expansion is commonly used to analyze the redshift-space power spectrum in the data analysis. The motivation for this expansion is that if one uses full angular information the Legendre moments are uncorrelated. Using Legendre polynomials $\mathcal{P}_{l}(\mu)$, we have

$$
P^{s}(\mathbf{k})=\sum_{l=0,2,4, \cdots} P_{l}^{s}(k) \mathcal{P}_{l}(\mu)
$$

The multipole moments, $P_{l}^{s}$, are obtained by inversion of this relation,

$$
P_{l}^{s}(k)=(2 l+1) \int_{0}^{1} P^{s}(\mathbf{k}) \mathcal{P}_{l}(\mu) d \mu .
$$

The errors rapidly grow with the order of the multipole. The combination of the monopole $(l=0)$, quadrupole $(l=2)$, and hexadecapole $(l=4)$ has almost the equivalent cosmological information to the full $2 \mathrm{D}$ spectrum, as argued by [52].

The difference between the two expansions presented above is just how to expand the redshiftspace power spectrum $P^{s}$, so they are equivalent if one considers infinite order terms. They are related to each other through a simple linear transform, as

$$
\left(\begin{array}{c}
P_{0}^{s}(k) \\
P_{2}^{s}(k) \\
P_{4}^{s}(k) \\
P_{6}^{s}(k) \\
P_{8}^{s}(k) \\
\vdots
\end{array}\right)=\left(\begin{array}{cccccc}
1 & 1 / 3 & 1 / 5 & 1 / 7 & 1 / 9 & \cdots \\
0 & 2 / 3 & 4 / 7 & 11 / 21 & 40 / 99 & \cdots \\
0 & 0 & 8 / 35 & 24 / 77 & 48 / 143 & \cdots \\
0 & 0 & 0 & 16 / 231 & 64 / 495 & \cdots \\
0 & 0 & 0 & 0 & 12 / 6435 & \cdots \\
\vdots & \vdots & \vdots & \vdots & \vdots & \ddots
\end{array}\right)\left(\begin{array}{c}
P_{\mu^{0}}(k) \\
P_{\mu^{2}}(k) \\
P_{\mu^{4}}(k) \\
P_{\mu^{6}}(k) \\
P_{\mu^{8}}(k) \\
\vdots
\end{array}\right)
$$

Thus, as addressed in [38], all terms will contribute to the monopole $l=0$, all except $P_{00}$ to quadrupole $l=2$, all but $P_{00}$ and $P_{01}$ to hexadecapole $l=4$, and so on.

\section{$3 \quad N$-body simulations}

\subsection{Dark matter halo and galaxy catalogs}

The power spectra of the derivative expansion are all from number-weighted velocity moments and thus can be straightforwardly measured from simulations. As in [49], we use a series of $N$-body simulations of the $\Lambda$ CDM cosmology seeded with Gaussian initial conditions, which is an updated version of [53]. The primordial density field is generated using the matter transfer function by CMBFAST [54]. We adopt the standard $\Lambda$ CDM model with the mass density parameter $\Omega_{m}=0.279$, the baryon density parameter $\Omega_{b}=0.0462$, the Hubble constant $h=0.7$, the spectral index $n_{s}=0.96$, and a normalization of the curvature perturbations $\Delta_{\mathcal{R}}^{2}=2.21 \times 10^{-9}$ (at $k=0.02 \mathrm{Mpc}^{-1}$ ) which gives the density fluctuation amplitude $\sigma_{8} \approx 0.807$, which are the best-fit parameters in the WMAP 5-year data [55]. We employ $1024^{3}$ particles of mass $m_{p}=2.95 \times 10^{11} h^{-1} M_{\odot}$ in a cubic box of side $1600 h^{-1} \mathrm{Mpc}$. The positions and velocities of all the dark matter particles are output at $z=0,0.509,0.989$, and 2.070, which are quoted as $z=0,0.5,1$, and 2 in what follows for simplicity. We use 12 independent realizations in order to reduce the statistical scatters.

Dark matter halos are identified at the four redshifts using the friends-of-friends algorithm with a linking length equal to 0.17 times the mean particle separation. We use all the halos with equal to or more than 20 particles. In order to investigate the halo mass dependences of the clustering 


\begin{tabular}{c|cccccc}
$z$ & $\begin{array}{c}\text { Mass } \\
\text { bin }\end{array}$ & $\begin{array}{c}\text { Mass range } \\
\left(10^{12} h^{-1} M_{\odot}\right)\end{array}$ & $\begin{array}{c}\bar{N} \\
\left(\times 10^{4}\right)\end{array}$ & $\begin{array}{c}\bar{n} \\
\left(h^{3} \mathrm{Mpc}^{-3}\right)\end{array}$ & $\begin{array}{c}b_{1}^{m h} \\
(\text { cross })\end{array}$ & $\begin{array}{c}b_{1}^{h h} \\
(\text { auto })\end{array}$ \\
\hline 0 & 1 & $5.91-17.7$ & 175 & $4.28 \times 10^{-4}$ & 1.17 & 1.19 \\
& 2 & $17.7-53.2$ & 63.3 & $1.54 \times 10^{-4}$ & 1.46 & 1.47 \\
& 3 & $53.2-159$ & 18.7 & $4.57 \times 10^{-5}$ & 2.03 & 1.99 \\
& 4 & $159-467$ & 4.05 & $9.89 \times 10^{-6}$ & 3.04 & 2.89 \\
\hline 0.5 & 1 & $5.91-17.7$ & 144 & $3.51 \times 10^{-4}$ & 1.64 & 1.65 \\
& 2 & $17.7-53.2$ & 44.8 & $1.09 \times 10^{-4}$ & 2.16 & 2.15 \\
& 3 & $53.2-159$ & 9.96 & $2.43 \times 10^{-5}$ & 3.12 & 3.04 \\
& 4 & $159-467$ & 1.30 & $3.18 \times 10^{-6}$ & 4.89 & 4.72 \\
& LRGs & $5.91-$ & 125 & $3.04 \times 10^{-4}$ & 2.16 & 2.15 \\
\hline 1 & 1 & $5.91-17.7$ & 101 & $2.46 \times 10^{-4}$ & 2.33 & 2.32 \\
& 2 & $17.7-53.2$ & 24.9 & $6.08 \times 10^{-5}$ & 3.18 & 3.16 \\
& 3 & $53.2-159$ & 3.68 & $8.98 \times 10^{-6}$ & 4.72 & 4.70 \\
\hline 2 & 1 & $5.91-17.7$ & 25.6 & $6.25 \times 10^{-5}$ & 4.65 & 4.73 \\
& 2 & $17.7-53.2$ & 2.94 & $7.18 \times 10^{-6}$ & 6.55 & 6.91
\end{tabular}

Table 1. Properties of halo catalogs. $\bar{N}$ and $\bar{n}$ are the number and number density of halos in each realization, respectively. The values $b_{1}^{m h}$ and $b_{1}^{h h}$ are the bias parameters computed from the cross $\left(P_{00}^{m h}\right)$ and auto $\left(P_{00}^{h h}\right)$ power spectra, respectively, averaged at $0.01 \leq k \leq 0.04 h \mathrm{Mpc}^{-1}$.

measurements, each dark matter halo catalog is divided into subsamples according to the halo mass, as $M_{i, \min } \leq M_{i} \leq M_{i, \max }$, where $M_{1, \min }=20 \times m_{p}$ and $M_{i+1, \min }=M_{i, \max }=3 M_{i, \min }$. Since the number density of halos is smaller at higher redshifts, we construct 4 halo subsamples at $z=0$ and $0.5,3$ subsamples at $z=1$, and 2 at $z=2$.

In order to analyze a more realistic sample, we use a halo occupation distribution (HOD) modeling which populates dark matter halos with galaxies according to the halo mass [e.g., 56-60]. We consider a luminous red galaxy (LRG) sample from the Baryon Oscillation Spectroscopic Survey (BOSS), which is part of Sloan Digital Sky Survey III (SDSS-III) [61,62]. Galaxies are assigned to the halos using the best fit HOD parameters for LRGs determined by [63] with the model of [64]. The fraction of satellite LRGs populated in our simulation samples is 12\%, consistent with [63]. For halos which contain satellite LRGs, we randomly pick up the same number of dark matter particles to represent the positions and velocities of the satellites. As we will see below, the correlation function measured from the mock LRG catalog is very consistent with that measured by [63] and [65]. Properties of the constructed halo and LRG catalogs are summarized in Table 1 .

\subsection{Power spectra and two-point correlation functions}

We measure the power spectra of dark matter halos and LRGs from our simulation samples following [49]. We assign the density field and the number-weighted velocity moments in real space on $1024^{3}$ grids using a cloud-in-cell interpolation method according to the positions of particles. To directly measure $P_{h h}^{s}(\mathbf{k})$ we also need the density field in redshift space. In measuring the redshift-space density field, we distort the positions of particles along the line-of-sight according to their peculiar velocities before we assign them to the grid. We regard each direction along the three axes of simulation boxes as the line of sight and the statistics are averaged over the three projections of all realizations for a total of 36 samples. We use a fast Fourier transform to measure the Fourier modes of the density fields in real space $\delta^{h}(\mathbf{k})$ and in redshift space $\delta_{s}^{h}(\mathbf{k})$, as well as the number-weighted velocity moment fields in real space $T_{\|}^{L, h}(\mathbf{k})$. Then the power spectrum in redshift space, $P_{h h}^{s}(\mathbf{k})$, as well as the power spectra of the number-weighted velocity moments $P_{L L^{\prime}}^{h h}(\mathbf{k})$, are measured by multiplying the modes of the two fields (or squaring in case of auto-correlation) and averaging over the modes within a bin. To obtain the final estimation of the auto-power spectrum in real space $P_{h h}^{r}$ and in redshift space $P_{h h}^{s}$, shot noise needs to be subtracted from the measured power spectrum. We assume the Poisson model where the contribution of the shot-noise to the halo power spectrum is described by an inverse 


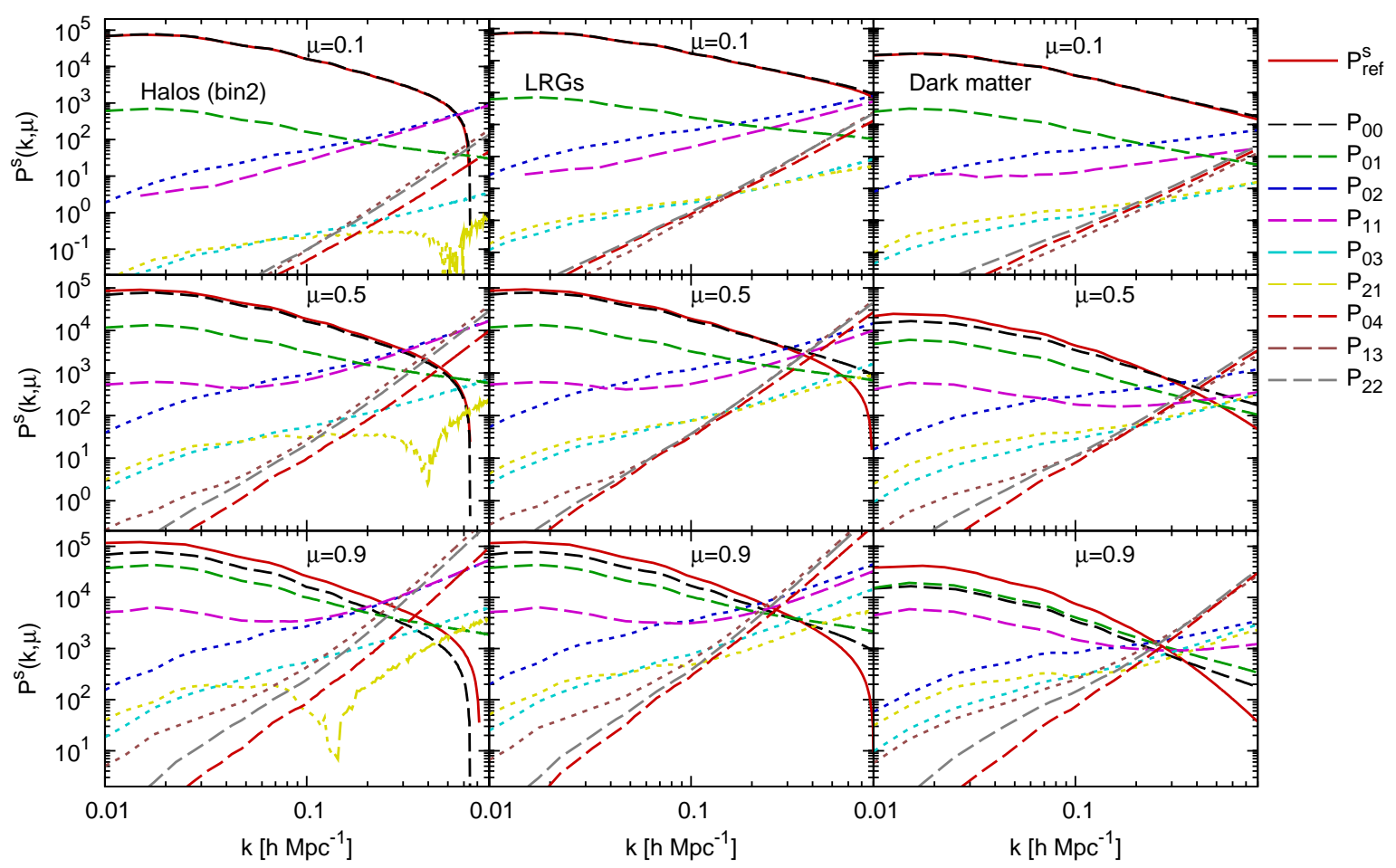

Figure 1. Power spectra measured in redshift space $P_{\text {ref }}^{s}(k, \mu)$ and individual contributions to it from the terms of the moments expansion up to 4 -th order at $z=0.5$ for halos (left), LRGs (middle) and dark matter (right). The halo subsample has almost the same bias value as the LRG sample. The width of $\mu$ bin is 0.2 , centered around the values shown in each panel. The dashed lines show the positive values while the dotted lines negative values.

of the halo number density. Error bars in the following results show the standard error on the mean. The dispersion in power spectra measurements is large on large scales because of sampling variance [66], but it is mostly eliminated by taking the ratio of the two spectra obtained from the same set of realizations [67-69].

Although we mainly analyze the power spectrum in this paper, its Fourier counter part, the two-point correlation function in redshift space $\xi_{h h}^{s}(\mathbf{s})$ is also presented for comparison. We measure the correlation function using the direct pair counting in configuration space.

\section{Numerical analysis}

\subsection{Power spectra of dark matter halos and galaxies}

We begin by presenting the redshift-space power spectra of halos and LRGs, $P_{h h}^{s}(\mathbf{k})$, directly measured in redshift space. The power spectrum in redshift space is shown as functions of $(k, \mu)$ at $z=0.5$ in figure 1. The results are shown for halos and LRGs at the left and middle columns, respectively. The mass range of the halo catalog used here is chosen to have $M_{h} \sim 2.84 \times 10^{13} h^{-1} M_{\odot}$ to have the same bias as LRGs. For comparison, the result for the dark matter, computed in [49], is shown in the right column. In figure 1 we also show contributions of the terms of $P_{L L^{\prime}}$ for $\left(0 \leq L+L^{\prime} \leq 4\right)$ to $P^{s}(k, \mu)$, computed from the number density-weighted velocity moments of halos and LRGs. The measurement of the angle-averaged power spectrum of momentum, $P_{11}(k)$, was presented in [70, 71].

Overall behavior of the power spectra for halos and LRGs is similar to the dark matter. At $\mu \sim 0$, contributions from the higher order power spectra of the velocity moments are small and $P^{s} \simeq P_{00}$, because each $P_{L L^{\prime}}$ is multiplied by a factor of $(k \mu)^{L+L^{\prime}}$. On large scales one expects $P_{00}$ to be followed by the other two linear order terms, which are $P_{01}$ and the scalar part of $P_{11}$. The correlators at the 
same order in powers of velocity, i.e. equal $L+L^{\prime}$, contain nontrivial cancellations among them [38]: higher-order $P_{L L^{\prime}}$ contain a shot noise term given by $\bar{n}^{-1}\left\langle u_{\|}^{L+L^{\prime}}\right\rangle$, which cancels out with other terms of the same order when the total contribution to the redshift-space power spectrum is considered. For example, in figure $1 P_{11}$ and $P_{02}$ have the similar amplitude but opposite signs, at high $k$. We do not see this for dark matter particles which have a much higher number density than halos and LRGs.

\subsection{Biasing of moments correlators}

For density fluctuations we define bias as the ratio of the power spectrum of biased objects to that of matter, $b^{2}(k)=P_{00}^{h h}(k) / P_{00}^{m}(k)$, with the shot noise subtracted from the halo spectrum $P_{00}^{h h}$. Following [38], we can generalize the concept of the bias to

$$
b_{L L^{\prime}}^{h h}(\mathbf{k})=\frac{P_{L L^{\prime}}^{h h}(\mathbf{k})}{P_{L L^{\prime}}^{m m}(\mathbf{k})} .
$$

On sufficiently large scales where linear theory is believed to be applicable, we have $b_{00}^{h h}=b_{1}^{2}, b_{01}^{h h}=b_{1}$ and $b_{11}^{h h}=1$, independent of scale and angle. All of the bias terms can be alternatively defined using the cross power spectrum, for example we can define $b_{00}^{m h}$ as

$$
\left(b_{00}^{m h}(k)\right)^{1 / 2}=\frac{P_{00}^{m h}(k)}{P_{00}^{m m}(k)},
$$

which is free from the shot noise issues. At large scales where the linear theory holds, $b_{00}^{h h}=b_{00}^{m h}$. Since we do not expect shot noise to be an issue for higher order moments correlators (as discussed in more detail below) we only look at the density-density correlations using the cross-correlations with the dark matter.

Here we wish to investigate the scale dependence of these generalized bias parameters. Figure 2 shows the halo bias defined above and determined from simulations. The top panels show $\left(b_{00}^{h h}\right)^{1 / 2}(k)$, shot noise corrected (eq. (4.1) with $\left.L=L^{\prime}=0\right)$, and $\left(b_{00}^{m h}(k)\right)^{1 / 2}$, the bias from the halo-matter crosscorrelation (eq. (4.2)). One can see that the two bias parameters agree on large scales and for low halo mass, corresponding to high halo number density. The more massive halos are more sparse, the shot noise correction becomes larger, and there is a difference between the two halo bias estimates even on very large scales. In addition, the bias estimated from the auto-correlation is strongly suppressed on small scales due to the shot noise correction (e.g. [72]). We assume the bias to be constant on sufficiently large scales and determine the large-scale bias $b_{1}^{m h}$ and $b_{1}^{\text {hh }}$ averaging the data points over $0.01 \leq k \leq 0.04 h \mathrm{Mpc}^{-1}$, shown in Table 1. We will use bias from cross-correlation as the true bias, assuming that the bias from auto-correlation suffers from imperfect shot noise subtraction.

Let us consider the next-order bias, $b_{01}^{h h}$, defined as

$$
b_{01}^{h h}(k)=\frac{P_{01}^{h h}(\mathbf{k})}{P_{01}^{m m}(\mathbf{k})}=\frac{P_{01}^{(2) h h}(k)}{P_{01}^{(2) m m}(k)} .
$$

Although $b_{L L^{\prime}}^{h h}$ has angular dependences by definition in general, $b_{01}^{h h}$ is a function of $k$ only as is well known for $b_{00}^{h h}$ and $b_{00}^{m h}$. In the second panels of figure 2, the bias parameter, $b_{01}^{h h}(k)$, is shown. In linear theory $b_{01}^{h h}=\left(b_{00}^{m h}\right)^{1 / 2}=b_{1}$, so $b_{01}^{h h}=\left(b_{00}^{m h}\right)^{1 / 2}$ with a percent level accuracy on sufficiently large scales, as expected. However, we also see a strong scale dependence of this bias, which is worse for the more biased halos.

For the next-order bias, $b_{11}^{h h}$, we need to separate the $\mu^{2}$ and $\mu^{4}$ parts. As detailed in [38], the $\mu^{2}$ part originates from the auto-correlation of the vector part of the momentum density, $P_{11}^{(2)}=P_{1,1}^{1,1,1}$. It is a nonlinear term, since vector component of momentum density is zero in linear theory. The $\mu^{4}$ term contains a linear order contribution, which is often described as the velocity auto-correlation. Thus we define $b_{11}^{h h}$ using the anisotropic term of $P_{11}$ as

$$
b_{11}^{h h}(k)=\frac{P_{11}^{(4) h h}(k)}{P_{11}^{(4) m m}(k)} .
$$




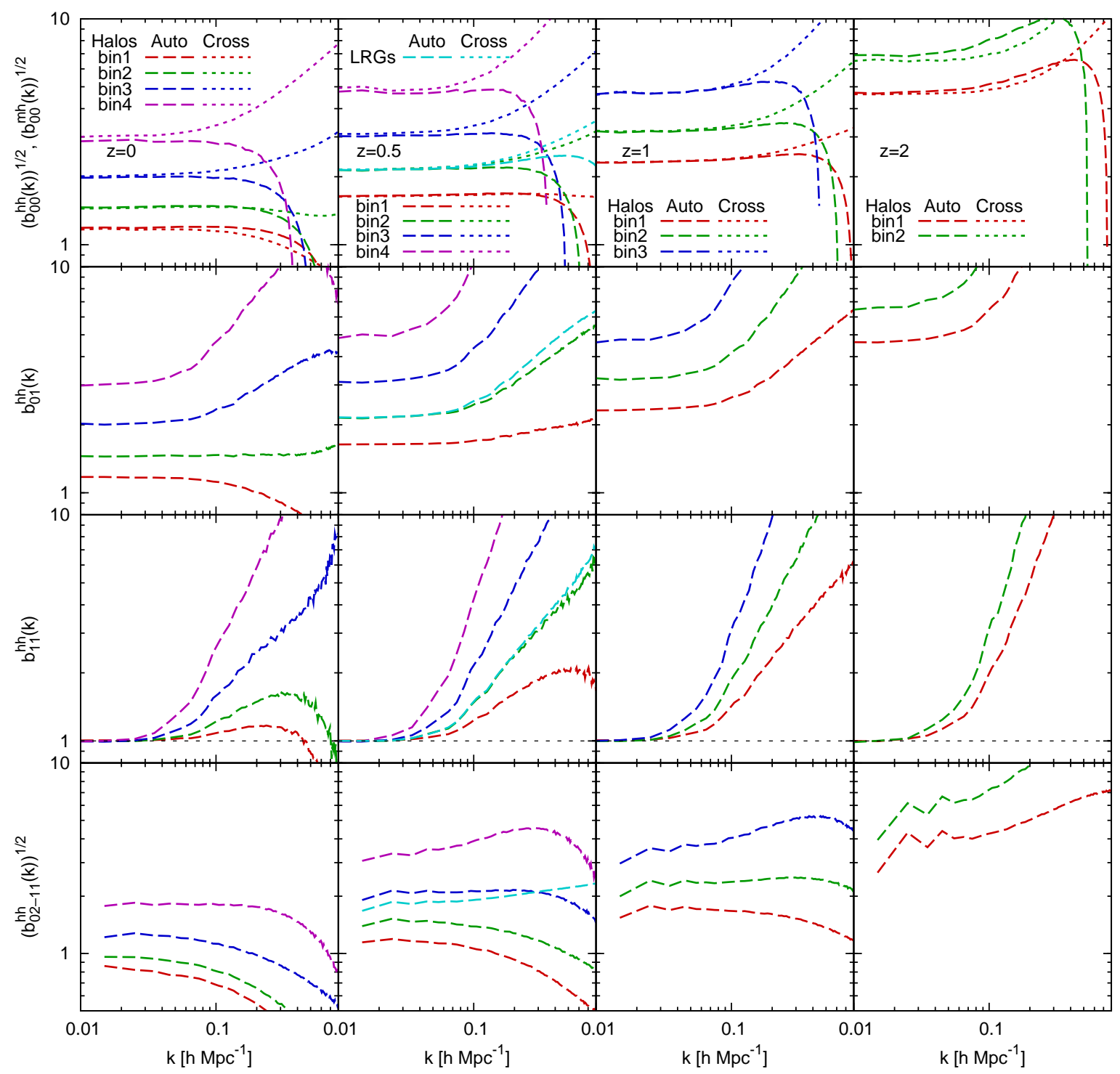

Figure 2. Bias parameters, $\left(b_{00}^{h h}\right)^{1 / 2}(k)$ and $\left(b_{00}^{m h}\right)^{1 / 2}(k)$ (top), $b_{01}^{h h}(k)$ (second), $b_{11}^{h h}(k)$ (third), and $b_{02-11}^{h h}(k)$ (bottom) for halos and LRGs. The light blue lines at $z=0.5$ show the results for LRGs, while all the other lines for halos of different mass bins. In the top panels the bias parameters computed using the auto $\left(P_{00}^{h}\right)$ and cross $\left(P_{00}^{m h}\right)$ power spectra are plotted as the dashed and dotted lines, respectively.

The bias defined in this way has no angular dependence. The third panels of figure 2 show the bias $b_{11}^{h h}$. We see that $b_{11}^{h h}$ starts to deviate from linear theory predictions at lower $k$ than $b_{01}^{h h}$ or $b_{00}^{m h}$ and these deviations are more important for more massive halos. These effects are large: for moderately biased halos with $b=2$ they are nearly a factor of 2 at $k=0.1 h \mathrm{Mpc}^{-1}$ at $z=0$. It is obvious that halos do not measure velocity-velocity correlations except on very large scales.

At the next order we have $P_{02}$, which contributes to the $\mu^{2} \operatorname{term}\left(P_{02}^{(2)}\right)$ and the $\mu^{4}$ term $\left(P_{02}^{(4)}\right)$ in $P^{s}$. The more important $\mu^{2}$ term contains a shot noise contribution $\sigma_{v}^{2} / n$, where $\sigma_{v}^{2}$ is the velocity dispersion. $P_{11}^{(2)}$ also contains the same shot noise term which cancels out that of $P_{02}^{(2)}$, as discussed in section 4.1. Neither of these terms, nor any of the higher order terms, contain any linear order contributions, so we do not expect them to contain cosmologically useful information, but it is important to understand them to estimate the nonlinear effects. We will thus combine the two $\mu^{2}$ terms into a 


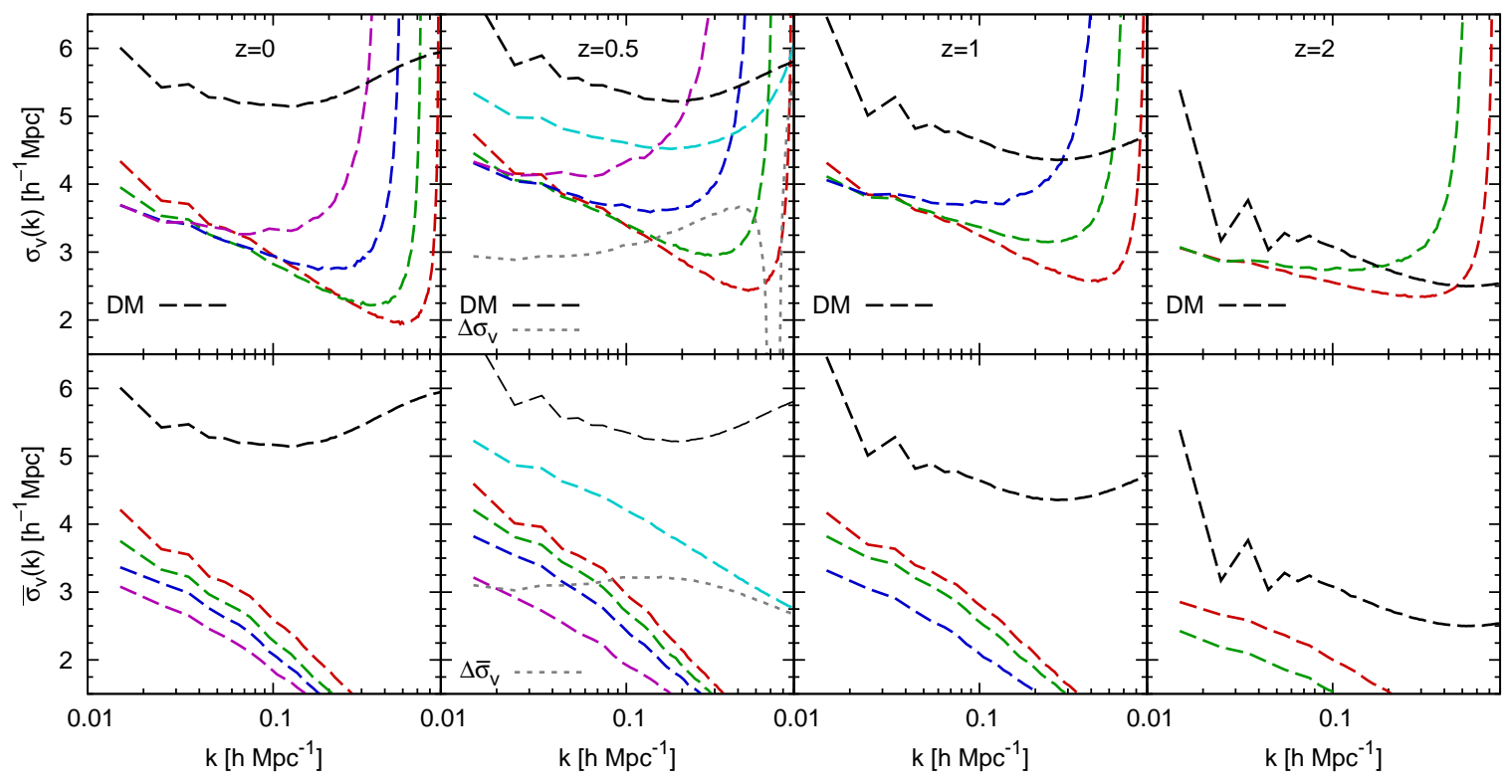

Figure 3. Velocity dispersion parameter with (upper panels) and without (lower panels) shot noise. The color of each line corresponds to the one with the same color in figure 2. The black line is for dark matter. The quantity $\Delta \sigma_{v}$ and $\Delta \bar{\sigma}_{v}$ are respectively shown in the top and bottom panels for $z=0.5$ as the dotted gray line (see the text).

bias term

$$
b_{02-11}^{h h}(k)=\frac{P_{02}^{(2) h h}(k)-P_{11}^{(2) h h}(k)}{P_{02}^{(2) m m}(k)-P_{11}^{(2) m m}(k)} .
$$

This is also shown in figure 2. We see that this bias scales strongly with density bias $b_{00}^{h} \sim b_{1}^{2}$.

Fingers-of-god (FoG) are caused by small scale velocity dispersion, which smears the galaxies along the line of sight and lead to a suppression of the linear terms in RSD power spectrum. The phenomenological model often used is that the linear terms, $P_{00}, P_{01}$ and $P_{11}$, are multiplied by FoG kernel which is often modeled as a Gaussian or a Lorentzian, and which is a function of $k^{2} \mu^{2} \sigma_{v}^{2}$, where $\sigma_{v}$ is the velocity dispersion. This velocity dispersion is sometimes modeled using linear theory averaged velocity squared $\left\langle v^{2}\right\rangle=\int P(k) d k / 2 \pi^{2}$, but as we show below this quantity is not relevant. The first term that is suppressed is $P_{00}$ and the leading order FoG term has a $k^{2} \mu^{2}$ dependence, $-k^{2} \mu^{2} \sigma_{v}^{2} P_{00}$. Since the only two terms besides $P_{01}$ leading to $\mu^{2}$ dependence in $P^{s}(k, \mu)$ are $P_{11}$ and $P_{02}$, and since these two terms each contain a shot noise that is canceled out in their total contribution to $P^{s}(k, \mu)$, it is natural to define the velocity dispersion as

$$
\sigma_{v}(k)=\sqrt{\frac{P_{02}^{(2) h h}(k)-P_{11}^{(2) h h}(k)}{P_{00}^{h h}(k)}} .
$$

The same quantity but with the shot noise included to the denominator $P_{00}^{h h}$ is denoted as $\bar{\sigma}_{v}(k)$. We show the velocity dispersion parameter of halos and LRGs with the shot noise subtracted from and included to $P_{00}^{h h}$ in the top and bottom of figure 3, respectively. For comparison, the velocity dispersion parameter computed for dark matter is also plotted at the both panels of the figure.

First thing to notice is that the velocity dispersion of halos is significantly smaller than that of dark matter or LRGs. This is to be expected, since halos have no small scale nonlinear velocity dispersion, as they are defined as the center of mass of the dark matter particles inside the halo. In contrast, LRG satellites and dark matter particles both stream around the halo center with high virial velocities inside the halos, which leads to a significant velocity dispersion. The velocity dispersion is nearly independent of the halo bias, as expected in the model above. 
Second thing to notice is that velocity dispersion of halos is rapidly decreasing towards smaller scales and has no relationship to $\left\langle v^{2}\right\rangle=\int P(k) d k / 2 \pi^{2} \sim(6 M p c / h)^{2}$ for this model at $z=0$. Physically this makes sense: any large scale bulk motions that contribute to $\left\langle v^{2}\right\rangle$ should have no effect on the relative velocity dispersion between two close particles, since they move both particles by the nearly same velocity. Thus there must be a significant reduction of velocity dispersion and FoG from bulk velocities as we go to higher $k$ and only the really small scale velocity dispersion from inside the halos can contribute to FoG on smaller scales. Even that small scale velocity dispersion must cancel out if one considers very high $k$, which is dominated by the zero lag correlations, i.e. the shot noise term. The exact cancellation between the shot noise of $P_{02}$ and $P_{11}$ is thus required [38]. This also shows that we must include $P_{11}$ in the FoG definition, since it partially cancels out $P_{02}$.

We can try to define the true small scale velocity dispersion of LRGs by using the halo sample which has the same bias as the LRGs at large scales, defining a quantity $\Delta \sigma_{v}$ as

$$
\Delta \sigma_{v}=\sqrt{\sigma_{v, \mathrm{LRG}}^{2}-\sigma_{v, h}^{2}},
$$

where $\sigma_{v, \mathrm{LRG}}$ is the velocity dispersion for LRGs and $\sigma_{v, h}$ is that for halos with the same bias as LRGs. The same quantity which includes the shot noise to $P_{00}^{h h}, \Delta \bar{\sigma}_{v}$, can be defined as well. The resulting $\Delta \sigma_{v}$ and $\Delta \bar{\sigma}_{v}$ are respectively shown at the top and bottom of figure 3 for $z=0.5$ as the dotted gray line. The difference of the velocity dispersion between LRGs and halos is nearly constant at $k<0.1 \mathrm{~h} \mathrm{Mpc}^{-1}$ : this is expected, since we argued that small scale velocity dispersion does not get canceled as quickly as the bulk motions. At higher $k$ even the small scale velocity dispersion cancels out.

\subsection{Relation to linear RSD}

In previous subsection we defined the bias of the velocity moment correlators relative to the dark matter. In order to investigate the ability of RSD to recover linear theory predictions we can also define the generalized halo bias alternatively for $\left(L L^{\prime}\right)=(0,0),(0,1)$ and $(1,1)$ as

$$
b_{L L^{\prime}, \operatorname{lin}}^{h h}(\mathbf{k})=\frac{P_{L L^{\prime}}^{h h}(\mathbf{k})}{P_{L L^{\prime}, \operatorname{lin}}^{m m}(\mathbf{k})},
$$

and the bias using the matter-halo cross power spectrum as

$$
\left(b_{00}^{m h}(k)\right)^{1 / 2}=\frac{P_{00}^{m h}(k)}{P_{00, \operatorname{lin}}^{m m}(k)},
$$

where $P_{00, \operatorname{lin}}^{m m}(k)$ is simply the linear mass power spectrum, and its relation to $P_{01}^{m m}$ and $P_{11}^{m m}$ is given in equation (2.8). These bias parameters based on the linear power spectrum for dark matter are shown in figure 4. We also show the nonlinear dark matter power spectrum relative to its linear spectrum as the black dotted curve. It is an auto correlation of dark matter thus defined as $b_{L L^{\prime}, \operatorname{lin}}^{m m}(k)=$ $P_{L L^{\prime}}^{m m}(k) / P_{L L^{\prime}, \text { lin }}^{m m}(k)$. Note that unlike the bias parameters $b_{L L^{\prime}}^{h h}$ and $b_{L L^{\prime}}^{m h}$ presented in section 4.2, the parameters $b_{L L^{\prime}, \text { lin }}^{h h}$ and $b_{L L^{\prime} \text {,lin }}^{m h}$ suffer from sampling variance at large scales. In order to reduce the large scatter because of sampling variance at $k \leq 0.04 h \mathrm{Mpc}^{-1}$, we divide them by $b_{L L^{\prime} \text { lin }}^{m m}(k)$ measured at redshift $z=8.5$ where the density perturbation is known to be linear at such scales.

The top row of figure 4 show the density bias parameter, $\left(b_{00,1 i n}^{h h}\right)^{1 / 2}$ as well as the bias for dark matter, $\left(b_{00, \text { lin }}^{m m}\right)^{1 / 2}$, while in the second row we show $\left(b_{00, \text { lin }}^{m h}\right)^{1 / 2}$. As is clear from the plots of $\left(b_{00, \text { lin }}^{m m}\right)^{1 / 2}$, the nonlinearity of $P_{00}^{m m}$ starts to be significant at $k \simeq 0.1 h \mathrm{Mpc}^{-1}$ for $z=0$ and $k \simeq 0.2 h \mathrm{Mpc}^{-1}$ for $z=2$.

In third row of figure 4 we compare the normalized first-order bias $b_{01, \text { lin }}^{h h}$ to $\left(b_{00,1 i n}^{h h}\right)^{1 / 2}$ and in fourth row to $\left(b_{00, \text { lin }}^{m h}\right)^{1 / 2}$, where

$$
\frac{b_{01, \text { lin }}^{h h}(k)}{\left(b_{00, \text { lin }}^{m h}(k)\right)^{1 / 2}}=\frac{k P_{01}^{(2) h h}(k)}{\mathcal{H} f P_{00}^{m h}(k)} .
$$




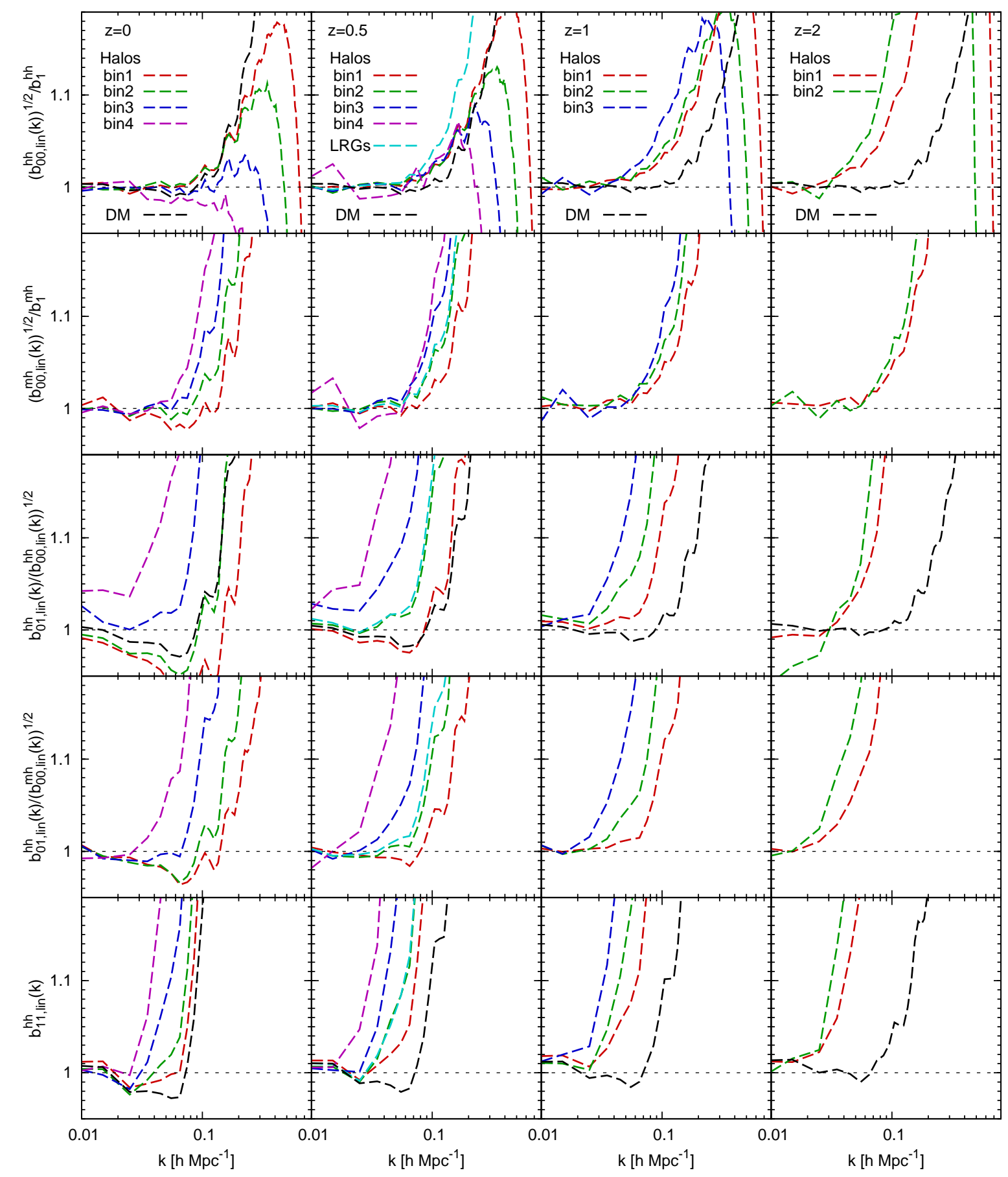

Figure 4. Same as figure 2, but the three power spectra which contain linear order contributions, $P_{00}^{m m}$, $P_{01}^{m m}$ and $P_{11}^{m m}$ in the definitions of bias parameters, are replaced by the linear theory power spectrum: from the top to bottom row, $\left(b_{00, \operatorname{lin}}^{h h}\right)^{1 / 2}(k) / b_{1}^{h h},\left(b_{00, \operatorname{lin}}^{m h}\right)^{1 / 2}(k) / b_{1}^{m h}, b_{01, \operatorname{lin}}^{h h}(k) /\left(b_{00}^{m h}\right)^{1 / 2}, b_{01, \operatorname{lin}}^{h h}(k) /\left(b_{00}^{h h}\right)^{1 / 2}$, and $b_{11, \text { lin }}^{h h}(k)$. The horizontal lines at the value of unity in each panel show the prediction for these quantities from linear theory with the input cosmological parameters in our simulations.

We see that for very low $k$ all the quantities, including the dark matter, are equal in the fourth row. This means that $P_{01}$ is tracing the true large scale halo bias as defined by the halo-matter 
cross-correlation. In contrast, the values in the third row differ at a level of a few percent, suggesting that the halo auto-power spectrum with the standard shot noise subtraction does not trace the true halo bias, most likely because the shot noise is not $\bar{n}^{-1}$, where $\bar{n}$ is the halo density. Since autocorrelation is measurable while cross-correlation with the dark matter is not (unless we have weak lensing measurements) this issue needs to be taken into account when analyzing real surveys. For LRGs the effect is below $1 \%$. In both rows we see that there is strong scale dependence of the bias: for LRGs it is $10 \%$ at $k=0.1 \mathrm{~h} \mathrm{Mpc}^{-1}$.

The next-order quantity relevant to the growth rate measurement is,

$$
b_{11, \operatorname{lin}}^{h h}(k)=\frac{k^{2} P_{11}^{(4) h h}(k)}{\mathcal{H}^{2} f^{2} P_{00, \operatorname{lin}}^{m m}(k)} .
$$

This term has $\mu^{4}$ angular dependence and dominates the hexadecapole. Although such a higher-order statistics is generally noisier than the lower-order statistics above, measuring $P_{11}$ has a potential to enable us to directly give constraints on $f$ thus on modified gravity models, independently from the galaxy biasing or the existence of the shot noise. We show the resulting parameter $b_{11, \text { lin }}^{\text {hh }}$ at the bottom of figure 4. At very low $k$ it approaches the true value, but the scale dependence is stronger than for $b_{01, l i n}^{h h}$ : only the lowest $k$ modes trace the dark matter velocity. For LRGs the nonlinear effect is $10 \%$ at $k=0.06 \mathrm{hpc}^{-1}$.

\subsection{Legendre moments of redshift-space power spectrum}

In this subsection we present the power spectrum directly measured in redshift space in section 4.1, $P^{s}(k, \mu)$, in terms of the Legendre multipole spectra. In the top set of figure 5 we show the resulting multipole spectra of mass-binned halos and LRGs. The top, middle and bottom panels respectively show the monopole, quadrupole and hexadecapole spectra. Monopole is shot noise subtracted using the standard shot noise $n^{-1}$. We show the corresponding linear theory predictions, $P_{l, \text { lin }}^{s}=b_{1} P_{l, \text { lin }}^{s, m}$. For the bias $b_{1}$ we use the values of $\left(b_{00}^{m h}(k)\right)^{1 / 2}$ (equation (4.2)) at large scales determined in section 4.2 and quoted in table 1 . The ratio to linear theory is shown in the bottom of figure 5 . As in the previous subsection, the effect of sampling variance on the ratio is prominent. We thus follow the same process: we divide the ratio of multipoles to linear theory by that computed at $z=8.5$ for the scales $k \leq 0.04 h \mathrm{Mpc}^{-1}$. Once again we see deviations from linear theory in the monopole on very large scales as a consequence of inaccurate shot noise subtraction in the auto-correlation. The halo monopole is typically first above the linear theory predicts due to the nonlinear and halo biasing effects, before it comes down because of the shot noise subtraction. In contrast, the dark matter monopole as measured in [49] is flat or decreased towards the smaller scales, shown at the bottom two rows of figure 5 as the dot-dashed lines.

The quadrupole moments show a similar behavior. They are not affected by the shot noise so there is no uncertainty with the exact value of the shot noise. As a result all of the halo quadrupole moments agree on vary large scales, similar to the situation in $P_{01}$. We note that because the quadrupole moment integrates over all the modes with a non-positive function $\mathcal{P}_{2}(\mu)=\left(3 \mu^{2}-1\right) / 2$, the result is a partial cancellation of modes and the quadrupole is more susceptible to the sampling variance: these effects reach $10 \%$ at $k \sim 0.02 \mathrm{~h} \mathrm{Mpc}^{-1}$. However, by dividing it by the quadrupole for dark matter at very high redshift, such sampling variance effects are well eliminated as shown on our plots. The quadrupole deviates from the linear theory prediction at larger scales than that for the monopole and typically the biasing and nonlinear effects make the halo quadrupole increase relative to the linear theory. In contrast, for the LRG sample and for the dark matter the effect is a strong suppression of quadrupole relative to the linear theory prediction. At the largest scales, the all the quadrupole spectra approach linear theory at $z=1$ and 2 , and just a few percent below at $z=0$ and $z=0.5$. We also show in figure 5 the hexadecapole. It is even noisier than the quadrupole [44] so we adopt $k$ binning for it twice as large as for the lower multipoles and put artificial cuts for the plot for $k<0.04 h \mathrm{Mpc}^{-1}$. 

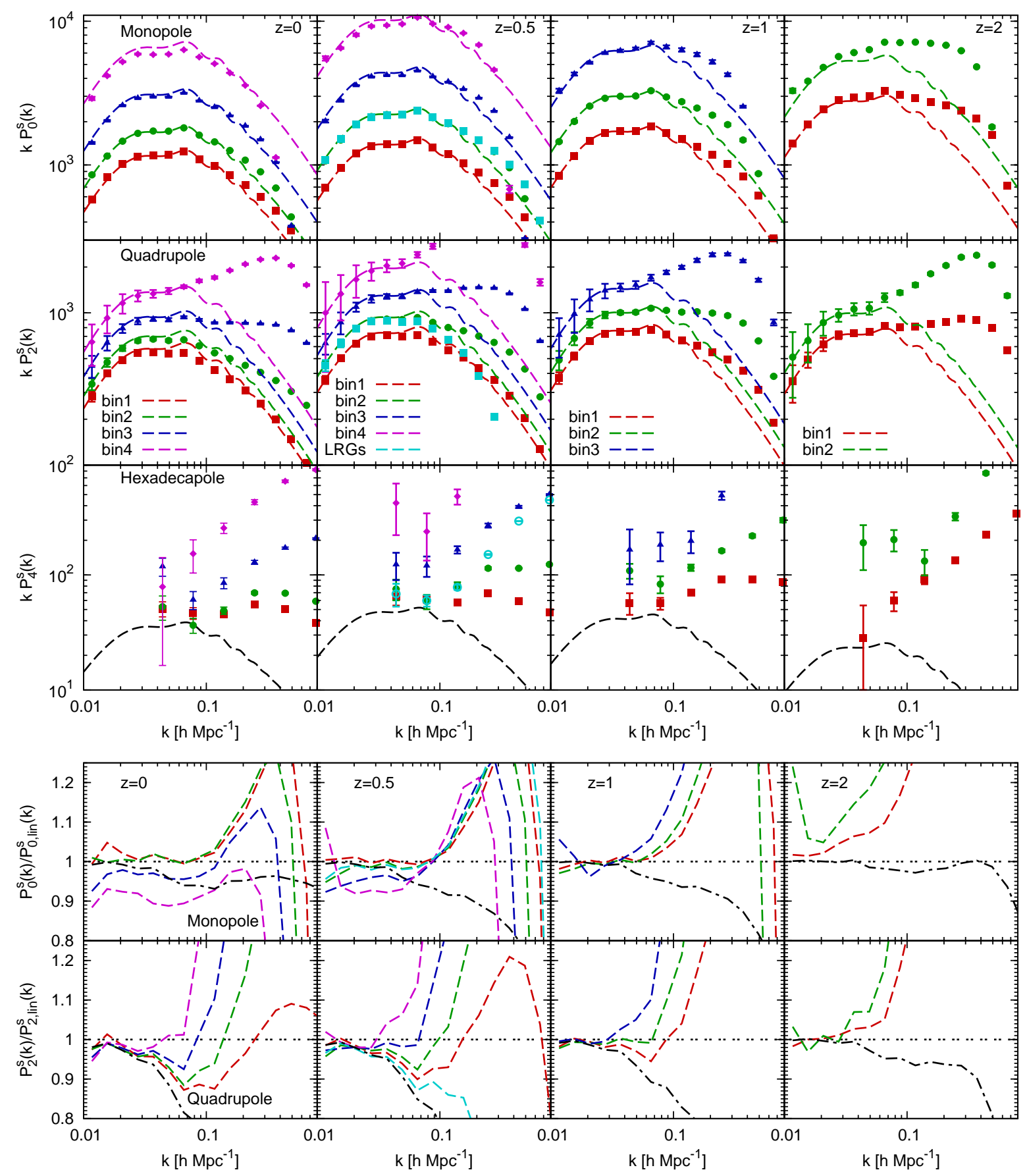

Figure 5. Top set: Multipole power spectra of halos and LRGs. The corresponding linear theory predictions are shown as the dashed line with the same color for monopoles and quadrupoles, while as the black line for hexadecapoles. Artificial cuts are put for the plots of the hexadecapoles at low $k$ because of large sampling variance. Bottom set: Monopole and quadrupole spectra divided by linear theory. The color of the lines corresponds to the one with the same color in the top set. The results for dark matter, obtained in [49], are also shown as the dot-dashed black lines for comparison. 


\section{Expansion in powers of $\mu^{2}$}

In previous work [49], using dark matter simulations, we have seen that the series expansion of equation 2.4 is convergent for $k \mu \sigma_{v} / \mathcal{H}<1$ but there is no convergence for $k \mu \sigma_{v} / \mathcal{H}>1$, where $\sigma_{v}$ is a typical rms velocity. This series expansion could be more convergent for halos than for dark matter since, as we have established, halo centers are not sensitive to the the velocity dispersion inside halos. The convergence is an issue if we want to investigate $P^{s}(k, \mu)$ or its Legendre moments. Alternatively, we can consider an expansion in powers of $\mu^{2}$, which enables us to sidestep the issue of divergence of the terms [38]: for any finite power of $\mu^{2}$ there is a finite number of $P_{L L^{\prime}}$ terms contributing to it. In [49] we tested the powers of $\mu^{2}$ expansion against the dark matter simulations. Here we want to apply this to halos and galaxies.

As is clear from equation (2.5), only the three lowest terms, $\mu^{0}, \mu^{2}$, and $\mu^{4}$, contain cosmological information at the linear order, so in principle these are the only relevant terms. However, if we expand the full $P^{s}(k, \mu)$ into powers of $\mu^{2}$ and try to determine the coefficients from the data, the resulting coefficients will be correlated: only Legendre expansion assures uncorrelated values. As a result there will be mixing of higher powers of $\mu^{2}$ into lower powers if they are not accounted for in the fits, or there will be strong degeneracies and the fits will be unstable if all the coefficients are accounted for but we allow them to take any value. At high $k$ it may be more advantageous to resum the higher $\mu^{2}$ terms into the so called FoG kernel [49] and fit for that instead.

In this section we determine the coefficients of $\mu^{2 j}$ for each $P_{L L^{\prime}}$. Because we know the exact angular dependence of each $P_{L L^{\prime}}$ 's [38] (see section 2.1), we treat the coefficients as free parameters and compute the $\chi^{2}$ statistics for the measurement of each $P_{L L^{\prime}}$. We present the results of the expansion in terms of $\mu^{2 j}$ and the contributions to them from each $P_{L L^{\prime}}$ up to 8th order in figures 6 9. Those for redshifts $z=0,0.5,1$ are shown in figures 6,7 and 8 , respectively. The results at $z=2$ for halos of bin1 are also shown in figure 8. We show the comparison of the results at $z=0.5$ for LRGs, dark matter and halos with the same bias as LRGs (bin2) in figure 9. We discuss the results in detail below.

\section{$5.1 \mu^{0}$ term}

The lowest order, $\mu^{0}$ term in the power spectrum is the real space power spectrum $P_{00}$. It is shown for dark matter, LRGs and halos with one mass bin at $z=0.5$ in figure 1 . The $\mu^{0}$ term has the dominant contribution to the monopole, as can be seen from figure 1 .

\section{$5.2 \quad \mu^{2}$ terms}

There are three terms contributing to the coefficient of the $\mu^{2}$ term in $P^{s}, P_{01}^{s}, P_{11}^{s}$ and $P_{02}^{s}$. At the top panels in figures $6-9$ we show the individual term contributions to the coefficient of the $\mu^{2}$ term in $P^{s}$ as well as the sum. The linear theory prediction of the term, $2 f b_{1}^{m h} P_{00, \operatorname{lin}}^{m}(k)$, is also plotted as black dot-dashed line, with $b_{1}^{m h}$ is a constant computed from the mass-halo cross power spectrum at large scales and shown in table 1 . For $\mu^{2} P_{01}^{s}$ dominates for low $k$, as that is the only term which does not vanish in linear theory. This term follows linear theory prediction for low $k$, while for $k>0.1 \mathrm{~h} \mathrm{Mpc}^{-1}$ it exceeds the linear theory, more so for the more biased halos.

This is partially canceled by $P_{11}^{s}+P_{02}^{s}$. The $P_{02}^{s}$ term is negative on small scales and the $P_{11}^{s}$ term is always positive, but the sum of the two is negative. As discussed above [38], the sum of the two cancels all the bulk motion contributions, including any possible shot noise. At the top panels of figures $6-9$ the sum of the $P_{11}^{s}$ and $P_{02}^{s}$ terms is shown for the coefficient of $\mu^{2}$. Although each of them has a high amplitude (figure 1), the sum for halos is suppressed and lower than that for dark matter because the velocity dispersion of halos is essentially zero. To see this cancellation more clearly, we show in the upper panels of figure 10 the total contributions to $\mu^{2}$ term divided by the dominant term $P_{01}$. In each case we have about $10 \%$ suppression of $P_{01}^{s}$ at $k \sim 0.1 h \mathrm{Mpc}^{-1}$.

As one can see in figure 9 , the total sum of the $\mu^{2}$ terms for the dark matter becomes negative due to a large $P_{02}^{s}$ caused by small scale velocity dispersion. As expected, the same behavior can be seen for LRGs in the second panels from the left in figure 9. We do not see such behaviors for halos 


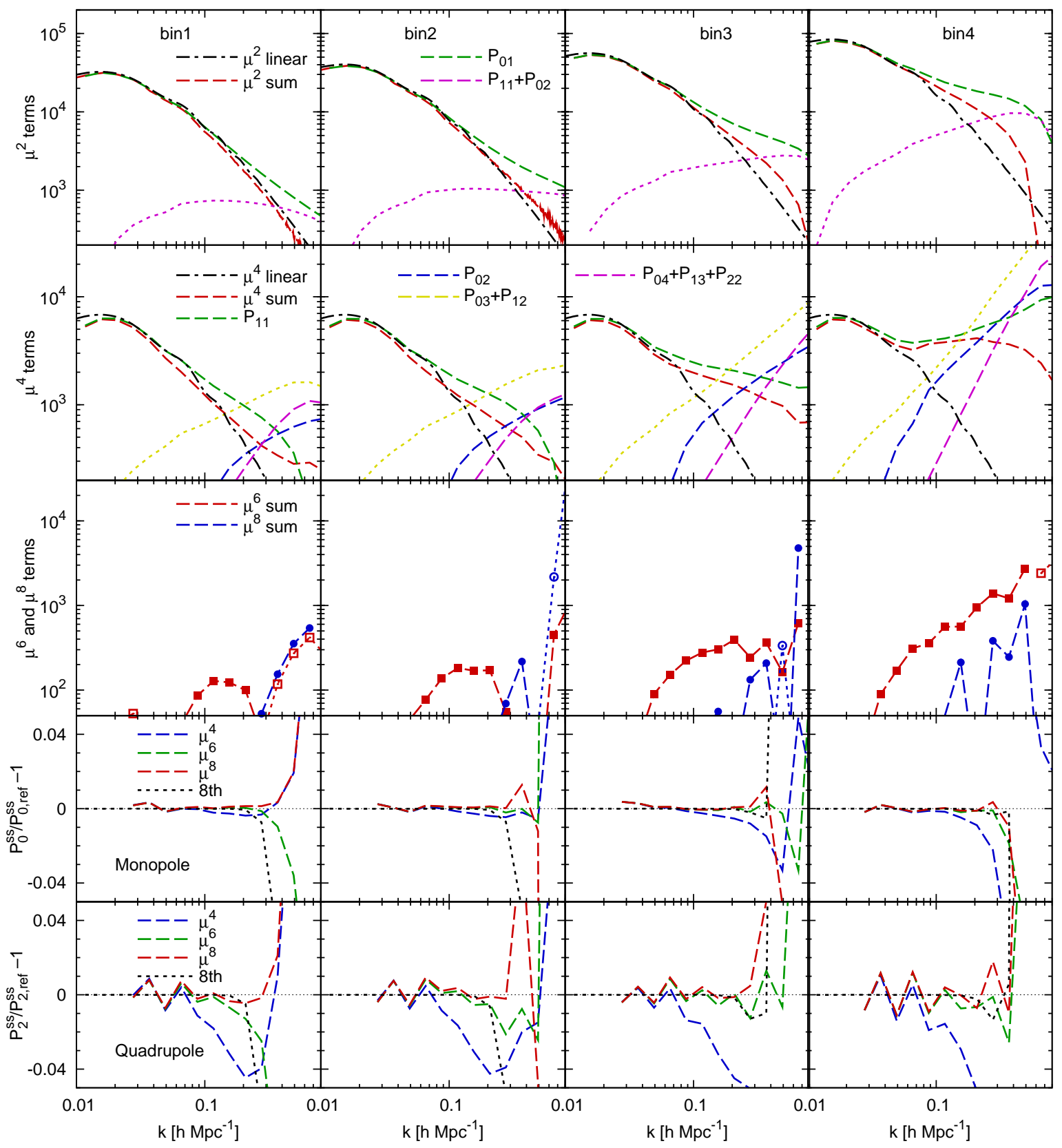

Figure 6. Contributions to $\mu^{2}$ (top), $\mu^{4}$ (second), and $\mu^{6}$ and $\mu^{8}$ (third) terms in redshift-space power spectrum $P^{s}(k, \mu)$ at $z=0$. The positive and negative contributions are shown as dashed and dotted lines, respectively, and additionally as the filled and open points for $\mu^{6}$ and $\mu^{8}$ terms. Linear theory predictions for $\mu^{2}$ and $\mu^{4}$ terms are shown as the dot-dashed curves. The bottom two rows show the error in the monopole and quadrupole as a function of order in powers of $\mu^{2}$ : up to $\mu^{4}$ (blue), $\mu^{6}$ (green) and $\mu^{8}$ (red) terms using equation (2.14). Because of large sampling variance, we put artificial cuts at large scales. Error between the summed power spectrum up to 8-th order and the reference spectrum is also shown as the dotted lines.

as in figures $6-8$. The result for halos with the same bias as LRGs at $z=0.5$ (bin2) is plotted again at the right panels of figure 9 for comparison. 


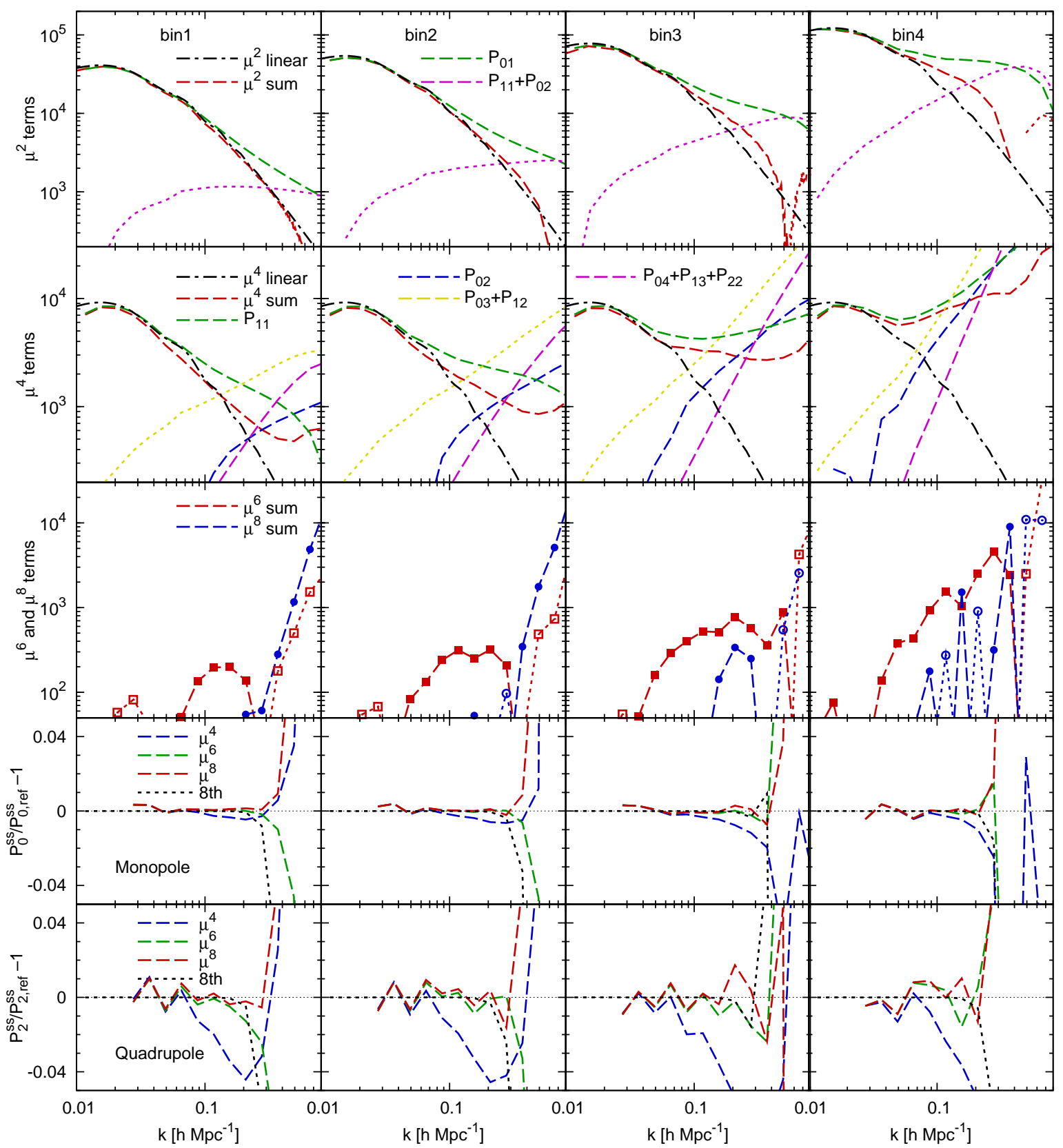

Figure 7. Same as figure 6 but for $z=0.5$. The results for LRGs are separately shown in figure 9.

\section{$5.3 \quad \mu^{4}$ terms}

The coefficient of the $\mu^{4}$ term in the power spectrum $P^{s}(k, \mu)$ contains contributions from 7 different terms, $P_{11}^{s}, P_{02}^{s}, P_{03}^{s}, P_{12}^{s}, P_{04}^{s}, P_{13}^{s}$ and $P_{22}^{s}$. They are shown in the second panels of figures $6-9$, together with the summation of the terms. $P_{11}^{s}$ is the only term which does not vanish in linear theory and thus dominates on large scales. This term approaches the linear theory prediction on large scales and is above that at small scales, just like in the case of $P_{00}$ in the $\mu^{0}$ term and $P_{01}$ in the $\mu^{2}$ term. Likewise, the deviation starts at larger scales and becomes more prominent for more biased halos. The lower panels of figure 10 show the summation of $\mu^{4}$ terms divided by the dominant term $P_{11}^{s}$. Even on the largest scales probed here $\left(k \sim 0.01 h \mathrm{Mpc}^{-1}\right)$, the higher-order terms do not vanish and 


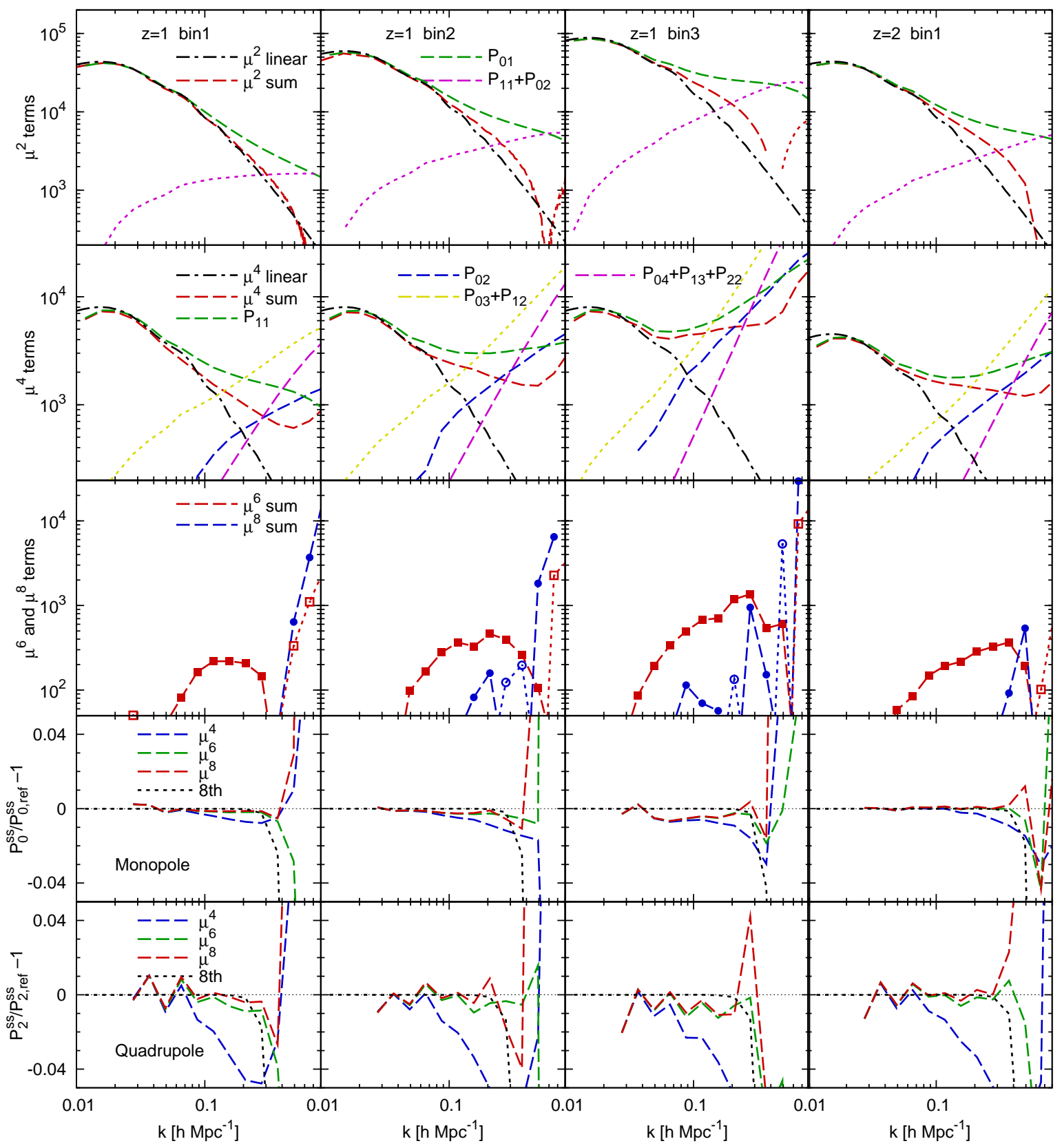

Figure 8. Same as figure 6 but for $z=1$ and $z=2$.

contribute a few percent.

The next order term in significance should be $P_{02}^{s}$. This term thus does not dominate at any scale, as we have also seen in dark matter clustering in [49] and can be seen in the left panels of figure 9. It is negative on all scales and act as suppression factors on the $\mu^{4}$ term in $P^{s}$. We have grouped the other terms together such that they cancel the each other's shot noise. The last order we need to consider for the $\mu^{4}$ term are the fourth order terms, $P_{04}, P_{22}$ and $P_{13}$. As discussed in [49] the bulk flow part of $P_{13}$ cancels out that of $P_{04}$ and $P_{22}$. They also have shot noise effects which are cancelled out when they are summed over. We thus show the total contribution from the three terms, $P_{04}+P_{13}+P_{22}$, in figures $6-9$. It adds power on small scales. Note that the contribution from the fourth order terms is smaller than that from the third order $P_{03}$ for halos, while it exceeds it at 


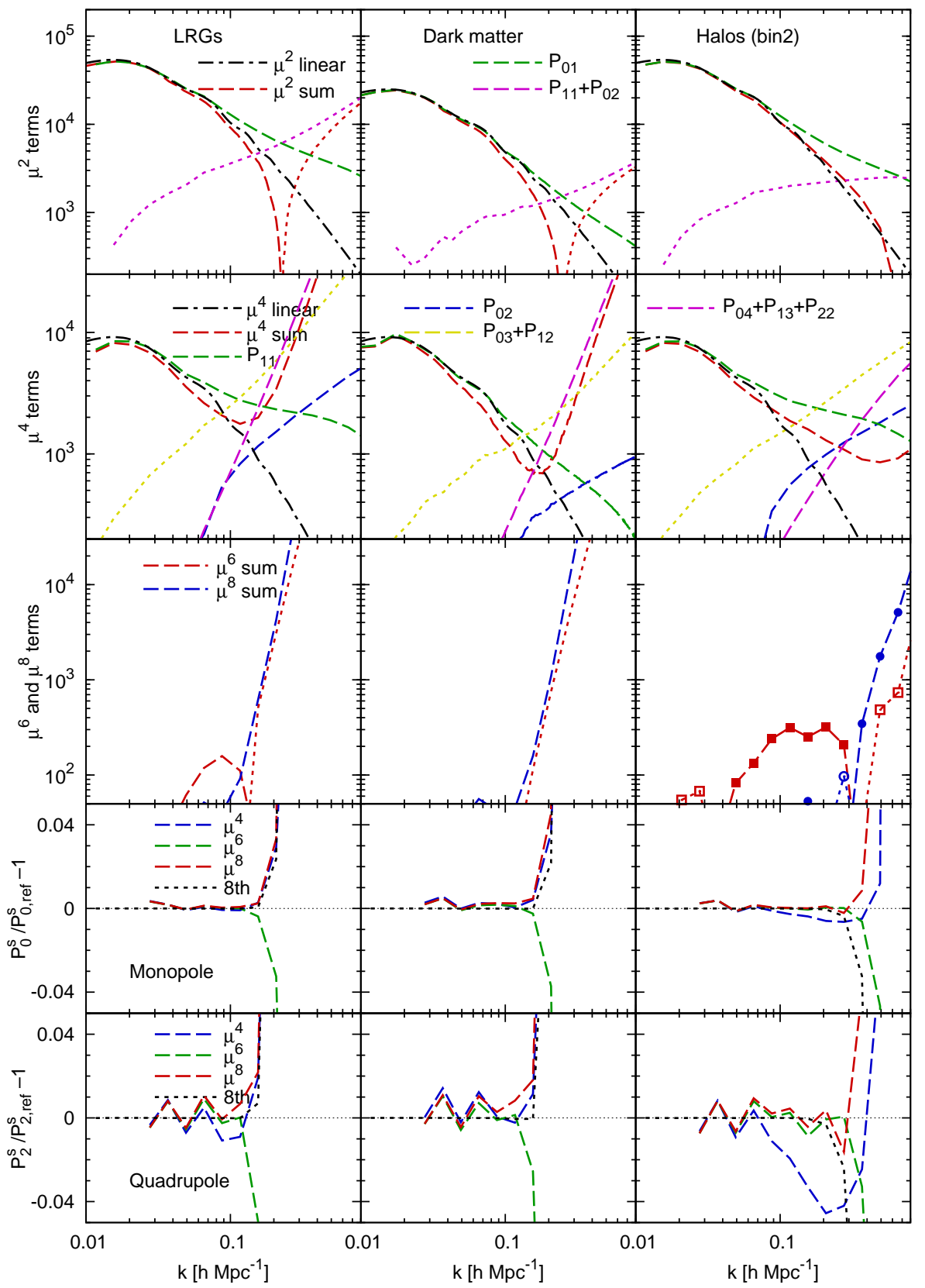

Figure 9. Same as figure 6 but at $z=0.5$ for LRGs (left) and dark matter (middle). The result for halos with the same bias as LRGs (bin2), already presented in figure 7, is shown again at the right panels for comparison.

$k>0.1 h \mathrm{Mpc}^{-1}$ for dark matter and LRGs because of the high random velocities.

\section{$5.4 \mu^{6}$ and $\mu^{8}$ terms}

At order higher than $\mu^{4}$ we do not have any linear order contributions, so these terms are expected to be small on large scales. There are many terms that contribute, third to sixth order terms in terms of $L+L^{\prime}$ to $\mu^{6}$ and fourth to eighth order terms to $\mu^{8}$. Third row in figures $6-9$ shows the total contribution from these terms to $\mu^{6}$ and $\mu^{8}$ terms. We can see that these terms are indeed negligibly small at large scales. At smaller scales these contributions increase with the scale dependences of $k^{6}$ 


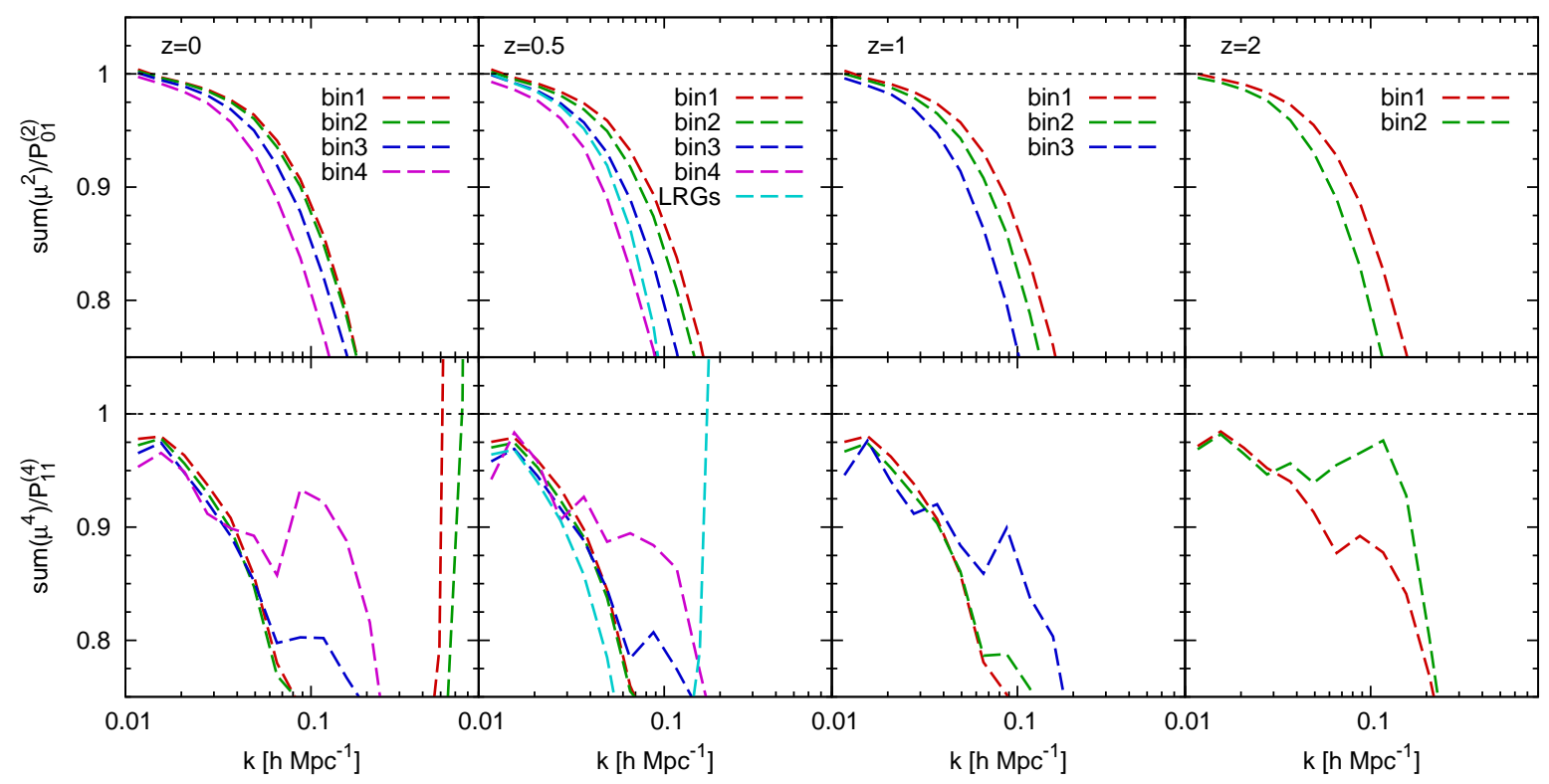

Figure 10. Contributions to $\mu^{2}$ (upper panels) and $\mu^{4}$ (lower panels) terms in $P^{s}$ divided by the dominant terms, $P_{01}^{(2)}$ and $P_{11}^{(4)}$, respectively.

and $k^{8}$, respectively. As $\mu^{4}$, the behaviors of $\mu^{6}$ and $\mu^{8}$ terms for LRGs are large and similar to the dark matter, a consequence of non-zero small scale velocity dispersion.

\subsection{Comparison of $\mu^{2}$ expansions with Legendre expansions}

It is worth studying how much is a given multipole moment affected by the expansion in powers of $\mu^{2}$ : we expect this expansion to break down at high $k$, while at low $k$ it should be strongly convergent. We transform the redshift-space power spectrum with $\mu^{j}$ expansions to Legendre moments using equation (2.14) and compare to moments measured directly in redshift space $P_{l, \text { ref }}^{s}$.

The error for the reconstructed monopole, $P_{0}^{s} / P_{0 \text {,ref }}^{s}-1$, is shown at the fourth row of figures $6-$ 9 , while that for the quadrupole, $P_{2}^{s} / P_{2, \text { ref }}^{s}-1$, is shown at the bottom row. The blue, green and red curves show the results when $\mu^{j}$ moments, $P_{\mu^{j}}$, are summed up to $\mu^{4}, \mu^{6}$ and $\mu^{8}$ terms, respectively in equation (2.14). One can see that the summation up to $\mu^{4}$ terms already has a good accuracy at $k \leq 0.4 h \mathrm{Mpc}^{-1}$ and $k \leq 0.2 h \mathrm{Mpc}^{-1}$ for the least massive and most massive halos at $z=0$, respectively. However, a more careful look at the figure reveals that the monopole estimated from the $\mu^{j}$ expansion up to $\mu^{4}$ terms starts to deviate from the reference spectrum at relatively large scales. The small deviation is eliminated by adding the $\mu^{6}$ and $\mu^{8}$ terms, which means these high-order nonlinear terms play an important role if one wants to predict the redshift-space power spectrum accurately. For the quadrupole moment, the true power spectrum cannot be reconstructed at $1 \%$ accuracy by using only the terms up to $\mu^{4}$ even at $k<0.1 \mathrm{~h} \mathrm{Mpc}^{-1}$, at least for $z=0$. Contributions from $\mu^{6}$ terms are necessary for the precise modeling of the quadrupole spectra. Adding $\mu^{8}$ terms further improves the accuracy. The deviations of the reconstructed quadrupole from the true one at large scales are due to the sampling variance.

For simulated LRGs at $z=0.5$ the convergence is worse: we find that the series breaks down already at $k \sim 0.1 h \mathrm{Mpc}^{-1}$, compared to $k \sim 0.2-0.3 h \mathrm{Mpc}^{-1}$ for the halos of the same bias and redshift. This is because small scale velocity dispersion inside halos increases the typical velocity. We also find that including terms above $\mu^{4}$ does not improve the convergence: a different approach, such as FoG resummation is needed in this case [49].

We also calculate the summed power spectrum, equation (2.4), which was analyzed for dark matter in detail by [49]. We show the accuracy of the summed power spectrum up to 8th order relative to the reference power spectrum, shown in the fifth and bottom rows of figures $6-9$ for the 

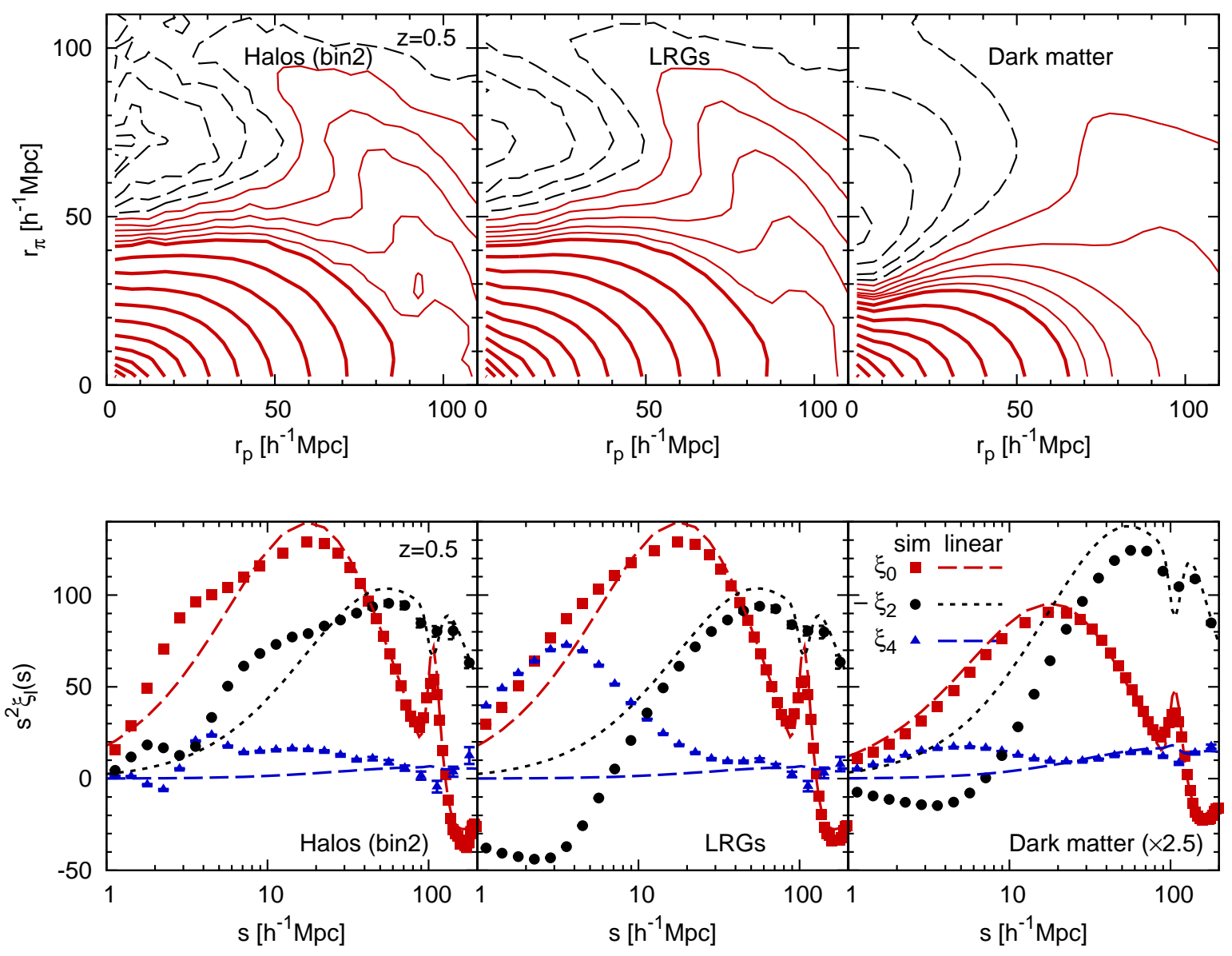

Figure 11. Top: 2D redshift-space correlation functions $\xi\left(r_{p}, r_{\pi}\right)$ at $z=0.5$. Bottom: multipoles of the correlation function. From left to right panels, we show the results for halos (bin2), LRGs, and dark matter. The amplitude of the multipoles of dark matter is multiplied by 2.5 for clarity.

monopole and quadrupole spectra, respectively. We see that the results are similar to the $\mu^{8}$ results, with no obvious advantage of one over the other.

\section{Configuration-space analysis}

So far our analysis was performed in Fourier space. In this section we present the redshift-space correlation function of halos and LRGs and compare to the power spectrum analysis presented in sections 4.1 and 4.4. Redshift-space correlation functions are computed as functions of separations perpendicular $\left(r_{p}\right)$ and parallel $\left(r_{\pi}\right)$ to the line of sight, $\xi^{s}\left(r_{p}, r_{\pi}\right)$. We show the 2D redshift-space correlation functions of dark matter, LRGs, and halos with the same bias as the LRGs, at $z=0.5$ at the top set of figure 11. Since anisotropy caused by the linear Kaiser effect is characterized by $\beta=f / b$ and $b=1$ for dark matter, the squashing along the line of sight is more prominent in dark matter clustering than in that of biased tracers. On the other hand, on smaller scales nonlinear random velocities smear the clustering along the line of sight, known as the FoG effect. Since dark matter and satellite LRGs have larger velocities, the correlation function at small scales is more elongated along the line of sight tan the halo correlation.

The two-dimensional correlation functions can be expanded in terms of Legendre multipole mo- 


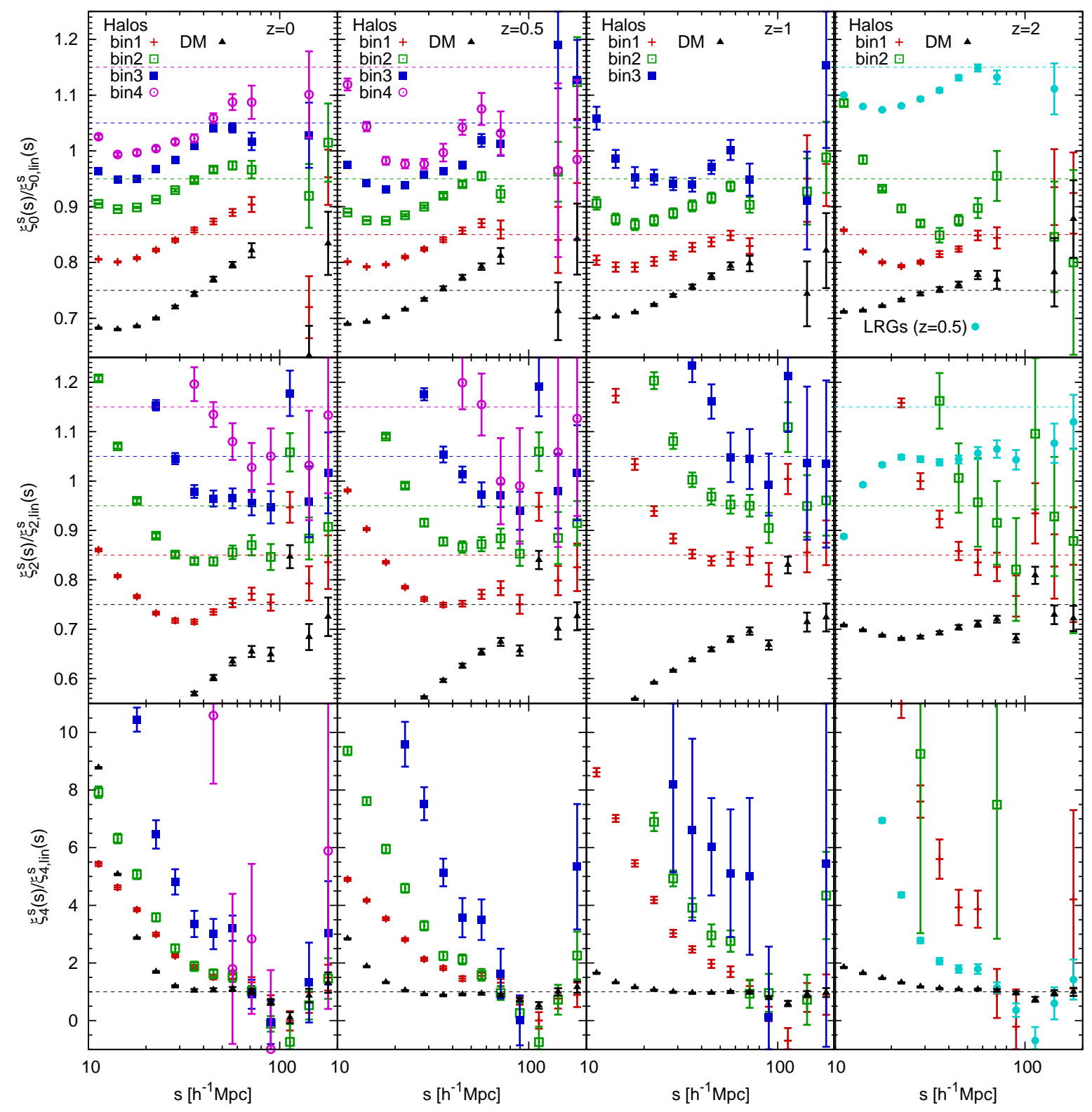

Figure 12. Ratios of multipoles to the corresponding linear theory predictions for dark matter, halos and LRGs. The results for all LRGs at $z=0.5$ are shown in the right panels, together with $z=2$ halos. For clarity the results of the monopole and quadrupole is offset by $-25 \%$ for dark matter, by $-15,-5,+5$ and $+15 \%$ for halos from the lightest to heaviest, and by $+15 \%$ for LRGs. Two data points of the monopoles at the separation around $s=100 h^{-1} \mathrm{Mpc}$ are not shown because the monopole correlation function crosses zero at such scales.

ments $\xi_{l}^{s}$, similarly to the power spectra, as

$$
\xi^{s}\left(r_{p}, r_{\pi}\right)=\sum_{l=0,2,4, \cdots} \xi_{l}^{s}(s) \mathcal{P}_{l}(\mu), \quad \xi_{l}^{s}(s)=(2 l+1) \int_{0}^{1} \xi^{s}\left(r_{p}, r_{\pi}\right) \mathcal{P}_{l}(\mu) d \mu
$$

where $\mu=r_{\pi} / s$. The multipole moments of the redshift-space correlation function, $\xi_{l}^{s}$, are related to 
the Fourier counterparts, $P_{l}^{s}$ (equation (2.13)) through

$$
\xi_{l}^{s}(s)=i^{l} \int \frac{d k k^{2}}{2 \pi^{2}} P_{l}^{s}(k) j_{l}(k s) .
$$

The three lowest-order multipoles which contain linear order contributions, the monopole, quadrupole, and hexadecapole, for the same three samples and the corresponding predictions from linear theory are shown at the bottom panels of figure 11. For the linear theory predictions for LRG and halos, the constant bias $b_{1}$ as measured in Fourier space using the cross-power spectrum (Table 1) has been used. The amplitude of the multipole moments for dark matter is multiplied by 2.5 for clarity. The monopole for the dark matter is below the linear theory, similar to the Fourier space result, while for LRGs and halos the monopole is above linear theory below $10 h^{-1} \mathrm{Mpc}$, also similar to the Fourier space analysis where the transition happens at $k \sim 0.1 h \mathrm{Mpc}^{-1}$.

The quadrupole for the dark matter deviates from the linear theory on a much larger scale, and becomes negative for $s<8 h^{-1}$ Mpc. Same happens for the LRG sample, which contains small scale nonlinear random velocities from satellites. The halo sample does not show this and the quadrupole is above the linear theory below $20 h^{-1} \mathrm{Mpc}$. The hexadecapole shows very large deviations from linear theory, as expected also from the Fourier analysis.

In figure 12 we show the multipoles of dark matter, halos and LRGs at all the four redshifts divided by the corresponding linear theory predictions for $10<s<200 h^{-1} \mathrm{Mpc}$. Because the monopole changes sign at $s \approx 130 h^{-1} \mathrm{Mpc}$ and the ratios with linear theory become noisy, we exclude two data points around the scale. The ratio of the monopole to the linear theory typically increases with scale above $10 h^{-1} \mathrm{Mpc}$, and is below the linear theory for $s<30-50 h^{-1} \mathrm{Mpc}$ $[19,43]$. The quadrupole for halos is below the corresponding linear theory prediction even on very large scales, by $10 \%$ at $s \simeq 90 h^{-1} \mathrm{Mpc}$. The quadrupole for dark matter and LRGs is suppressed compared to the linear theory on small scales due to virialized random velocities. The hexadecapole starts to deviate from the linear theory predictions at very large scales, more so for more biased halos [43]. The dark matter hexadecapole is also above the linear theory, but the amplitude of enhancement is much smaller. Overall the configuration space analysis is similar and consistent with the power spectrum analysis, however the nonlinear and/or scale dependent bias effects are even stronger and in some cases we do not converge to linear theory even on very large scales.

\section{Conclusions}

The promise of galaxy redshift surveys is that it contains much of the cosmological information needed to extract information on cosmological parameters such as the dark energy properties and neutrino mass. Redshift space distortions can provide important information, in the sense of tracing the dark matter velocity field, but can also damage it, in the sense of being responsible for nonlinear effects that spoil the comparison between observations and linear theory predictions.

In the phase-space distribution function approach redshift-space distortions (RSD) can be written as a sum over density-weighted velocity moment correlators [38]. In this paper we extend the previous work and test this approach to RSD of discrete objects such as dark matter halos and galaxies, which, unlike the dark matter, are the observables in redshift space. For this purpose we construct a large set of cosmological $N$-body simulations, dividing each dark matter halo catalog into the mass bins for redshifts $z=0,0.5,1$ and 2. As an example of a more realistic galaxy sample we construct a mock BOSS-type LRG sample by applying the HOD modeling to the simulated halos at $z=0.5$.

Because RSD can be expressed as a sum over number weighted velocity moment correlators, we can individually compute each correlator term in the expansion of the redshift-space power spectrum and compare to its dark matter analog. In doing so we thus construct a number of generalized bias parameters, of which the first three can also be compared to the linear theory predictions. We find that velocity moment correlators deviate from linear theory predictions on a scale larger than the densitydensity correlation, more so if the underlying tracer is strongly biased. This can be understood by the fact that the number or mass weighting of the velocity field in these moments gives rise to scale 
dependent bias effects even if the density bias itself is not scale dependent. These effects typically enhance RSD on small scales for biased tracers relative to that of the dark matter.

In addition to these biasing effects one must also include the higher correlators, which are often described as a Fingers of God effects. It is often assumed that RSD are suppressed due to these higher order effects, which can lead to smearing of galaxies in the radial direction. We find that this effect is small for halos, which are not sensitive to the small scale velocity dispersion, and as a result RSD in halos are enhanced at high wavenumber relative to linear theory predictions, contrary to the commonly assumed model. This changes once a more realistic galaxy sample with satellites is analyzed, since satellites provide small scale velocity dispersion, but the amplitude of the effect depends on how these galaxies are populated inside the halo and what is the satellite fraction. It may be possible to construct galaxy samples with a small satellite fraction for which the small scale velocity dispersion is negligible.

We explore a number of different statistics in this paper: the full 2-dimensional power spectrum $P^{s}(k, \mu)$, as well as its Legendre moments monopole, quadrupole and hexadecapole. These all receive an infinite number of velocity moment correlators and we explore the convergence. We also explore expansion in powers of $\mu^{2 j}$, each of which contains a finite number of terms for a given $j$. We have computed the coefficients of $\mu^{2 j}$ terms of dark matter halos and LRGs up to $\mu^{8}$. Finally, we also explore the configuration space statistics such as the monopole and quadrupole of the correlation function, finding good agreement with previous analyses [43].

In all cases we find that nonlinear and/or scale dependent bias effects are very large even on large scales, specially for biased tracers. For example, nonlinear and biasing effects for LRGs similar to those in SDSS are of the order of $10 \%$ at $k \sim 0.15 h \mathrm{Mpc}^{-1}$ for the monopole, $k \sim 0.07 h \mathrm{Mpc}^{-1}$ for the quadrupole and even smaller $k$ in the hexadecapole. This is a much larger scale (smaller $k$ ) than the often stated assumption that they are negligible up to $k \sim 0.1 h \mathrm{Mpc}^{-1}$. This makes a large difference in the amount of information on $f \sigma_{8}$ we can extract from RSD, since the signal scales as $k^{3 / 2}$, where $k$ is the maximum Fourier wavevector we can still model. Some of this can be restored with a nonlinear model of RSD, but most of the models proposed so far do not account for the scale dependent bias of $P_{01}$ and $P_{11}$ induced by the $b>1$ galaxies [33, 44]. We will present a perturbation theory based study of the nonlinear and biasing effects elsewhere (Vlah et. al. 2012, in preparation).

\section{Acknowledgments}

We would like to thank Pat McDonald, Zvonimir Vlah, Tobias Baldauf, and Beth Reid for useful discussions. This research was supported by the DOE, and the Swiss National Foundation under contract 200021-116696/1 and Republic of Korea WCU grant R32-10130. V.D. acknowledges support by the Swiss National Science Foundation.

\section{References}

[1] P. J. E. Peebles, The large-scale structure of the universe (1980).

[2] N. Kaiser, Mon. Not. Roy. Astron. Soc. 227, 1 (Jul. 1987).

[3] A. J. S. Hamilton, in D. Hamilton, ed., The Evolving Universe (1998), vol. 231 of Astrophysics and Space Science Library, pp. 185-+, arXiv:astro-ph/9708102.

[4] E. V. Linder, Phys. Rev. D 72(4), 043529 (Aug. 2005), arXiv:astro-ph/0507263.

[5] B. Jain and P. Zhang, Phys. Rev. D 78(6), 063503 (Sep. 2008), 0709.2375.

[6] L. Guzzo, M. Pierleoni, B. Meneux, E. Branchini, O. Le Fèvre, C. Marinoni, B. Garilli, J. Blaizot, G. De Lucia, A. Pollo, et al., Nature 451, 541 (Jan. 2008), 0802.1944.

[7] Y. Song and K. Koyama, JCAP 1, 48 (Jan. 2009), 0802.3897.

[8] J. A. Peacock, S. Cole, P. Norberg, C. M. Baugh, J. Bland-Hawthorn, T. Bridges, R. D. Cannon, M. Colless, C. Collins, W. Couch, et al., Nature 410, 169 (Mar. 2001), arXiv:astro-ph/0103143. 
[9] I. Zehavi, M. R. Blanton, J. A. Frieman, D. H. Weinberg, H. J. Mo, M. A. Strauss, S. F. Anderson, J. Annis, N. A. Bahcall, M. Bernardi, et al., Astrophys. J. 571, 172 (May 2002), arXiv:astro-ph/0106476.

[10] E. Hawkins, S. Maddox, S. Cole, O. Lahav, D. S. Madgwick, P. Norberg, J. A. Peacock, I. K. Baldry, C. M. Baugh, J. Bland-Hawthorn, et al., Mon. Not. Roy. Astron. Soc. 346, 78 (Nov. 2003), arXiv:astro-ph/0212375.

[11] M. Tegmark, M. A. Strauss, M. R. Blanton, K. Abazajian, S. Dodelson, H. Sandvik, X. Wang, D. H. Weinberg, I. Zehavi, N. A. Bahcall, et al., Phys. Rev. D 69(10), 103501 (May 2004), arXiv:astro-ph/0310723.

[12] M. Tegmark, D. J. Eisenstein, M. A. Strauss, D. H. Weinberg, M. R. Blanton, J. A. Frieman, M. Fukugita, J. E. Gunn, A. J. S. Hamilton, G. R. Knapp, et al., Phys. Rev. D 74(12), 123507 (Dec. 2006), arXiv:astro-ph/0608632.

[13] N. P. Ross, J. da Ângela, T. Shanks, D. A. Wake, R. D. Cannon, A. C. Edge, R. C. Nichol, P. J. Outram, M. Colless, W. J. Couch, et al., Mon. Not. Roy. Astron. Soc. 381, 573 (Oct. 2007), arXiv:astro-ph/0612400.

[14] T. Okumura, T. Matsubara, D. J. Eisenstein, I. Kayo, C. Hikage, A. S. Szalay, and D. P. Schneider, Astrophys. J. 676, 889 (Apr. 2008), 0711.3640.

[15] A. Cabré and E. Gaztañaga, Mon. Not. Roy. Astron. Soc. 393, 1183 (Mar. 2009), 0807.2460.

[16] C. Blake, S. Brough, M. Colless, C. Contreras, W. Couch, S. Croom, T. Davis, M. J. Drinkwater, K. Forster, D. Gilbank, et al., ArXiv e-prints (Apr. 2011), 1104.2948.

[17] J. L. Tinker, D. H. Weinberg, and Z. Zheng, Mon. Not. Roy. Astron. Soc. 368, 85 (May 2006), arXiv:astro-ph/0501029.

[18] V. Desjacques and R. K. Sheth, Phys. Rev. D 81(2), 023526 (Jan. 2010), 0909.4544.

[19] T. Okumura and Y. P. Jing, Astrophys. J. 726, 5 (Jan. 2011), 1004.3548.

[20] E. Jennings, C. M. Baugh, and S. Pascoli, Mon. Not. Roy. Astron. Soc. 410, 2081 (Jan. 2011), 1003.4282 .

[21] J. Kwan, G. F. Lewis, and E. V. Linder, ArXiv e-prints (May 2011), 1105.1194.

[22] F. Bernardeau, S. Colombi, E. Gaztañaga, and R. Scoccimarro, Phys. Rept. 367, 1 (Sep. 2002), arXiv:astro-ph/0112551.

[23] M. Crocce and R. Scoccimarro, Phys. Rev. D 73(6), 063519 (Mar. 2006), arXiv:astro-ph/0509418.

[24] M. Crocce and R. Scoccimarro, Phys. Rev. D 73(6), 063520 (Mar. 2006), arXiv:astro-ph/0509419.

[25] S. Matarrese and M. Pietroni, JCAP 6, 26 (Jun. 2007), arXiv:astro-ph/0703563.

[26] P. McDonald, Phys. Rev. D 75(4), 043514 (Feb. 2007), arXiv:astro-ph/0606028.

[27] P. Valageas, Astron. Astrophys. 465, 725 (Apr. 2007), arXiv:astro-ph/0611849.

[28] A. Taruya and T. Hiramatsu, Astrophys. J. 674, 617 (Feb. 2008), 0708.1367.

[29] A. F. Heavens, S. Matarrese, and L. Verde, Mon. Not. Roy. Astron. Soc. 301, 797 (Dec. 1998), arXiv:astro-ph/9808016.

[30] R. Scoccimarro, H. M. P. Couchman, and J. A. Frieman, Astrophys. J. 517, 531 (Jun. 1999), arXiv:astro-ph/9808305.

[31] S. Bharadwaj, Mon. Not. Roy. Astron. Soc. 327, 577 (Oct. 2001), arXiv:astro-ph/0105320.

[32] B. Pandey and S. Bharadwaj, Mon. Not. Roy. Astron. Soc. 358, 939 (Apr. 2005), arXiv:astro-ph/0403670.

[33] R. Scoccimarro, Phys. Rev. D 70(8), 083007 (Oct. 2004), arXiv:astro-ph/0407214.

[34] J. C. Jackson, Mon. Not. Roy. Astron. Soc. 156, 1P (1972).

[35] T. Matsubara, Phys. Rev. D 77(6), 063530 (Mar. 2008), 0711.2521.

[36] A. Taruya, T. Nishimichi, and S. Saito, Phys. Rev. D 82(6), 063522 (Sep. 2010), 1006.0699. 
[37] P. Valageas, Astron. Astrophys. 526, A67+ (Feb. 2011), 1009.0106.

[38] U. Seljak and P. McDonald, JCAP 11, 39 (Nov. 2011), 1109.1888.

[39] N. Kaiser, Astrophys. J. Let. 284, L9 (Sep. 1984).

[40] J. M. Bardeen, J. R. Bond, N. Kaiser, and A. S. Szalay, Astrophys. J. 304, 15 (May 1986).

[41] T. Matsubara, Phys. Rev. D 78(8), 083519 (Oct. 2008), 0807.1733.

[42] M. Sato and T. Matsubara, Phys. Rev. D 84(4), 043501 (Aug. 2011), 1105.5007.

[43] B. A. Reid and M. White, Mon. Not. Roy. Astron. Soc. 417, 1913 (Nov. 2011), 1105.4165.

[44] T. Nishimichi and A. Taruya, Phys. Rev. D 84(4), 043526 (Aug. 2011), 1106.4562.

[45] S. de la Torre and L. Guzzo, ArXiv e-prints (Feb. 2012), 1202.5559.

[46] P. Zhang, M. Liguori, R. Bean, and S. Dodelson, Physical Review Letters 99(14), 141302 (Oct. 2007), 0704.1932.

[47] R. Reyes, R. Mandelbaum, U. Seljak, T. Baldauf, J. E. Gunn, L. Lombriser, and R. E. Smith, Nature 464, 256 (Mar. 2010), 1003.2185.

[48] C. Hikage, M. Takada, and D. N. Spergel, Mon. Not. Roy. Astron. Soc. 419, 3457 (Feb. 2012), 1106.1640.

[49] T. Okumura, U. Seljak, P. McDonald, and V. Desjacques, JCAP 2, 10 (Feb. 2012), 1109.1609.

[50] Z. Vlah, U. Seljak, P. McDonald, T. Okumura, and T. Baldauf, in preparation (2012).

[51] P. McDonald, JCAP 4, 32 (Apr. 2011), 0910.1002.

[52] A. Taruya, S. Saito, and T. Nishimichi, Phys. Rev. D 83(10), 103527 (May 2011), 1101.4723.

[53] V. Desjacques, U. Seljak, and I. T. Iliev, Mon. Not. Roy. Astron. Soc. 396, 85 (Jun. 2009), 0811.2748.

[54] U. Seljak and M. Zaldarriaga, Astrophys. J. 469, 437 (Oct. 1996), arXiv:astro-ph/9603033.

[55] E. Komatsu, J. Dunkley, M. R. Nolta, C. L. Bennett, B. Gold, G. Hinshaw, N. Jarosik, D. Larson, M. Limon, L. Page, et al., Astrophys. J. Suppl. 180, 330 (Feb. 2009), 0803.0547.

[56] Y. P. Jing, H. J. Mo, and G. Boerner, Astrophys. J. 494, 1 (Feb. 1998), arXiv:astro-ph/9707106.

[57] U. Seljak, Mon. Not. Roy. Astron. Soc. 318, 203 (Oct. 2000), arXiv:astro-ph/0001493.

[58] R. Scoccimarro, R. K. Sheth, L. Hui, and B. Jain, Astrophys. J. 546, 20 (Jan. 2001), arXiv:astro-ph/0006319.

[59] A. A. Berlind and D. H. Weinberg, Astrophys. J. 575, 587 (Aug. 2002), arXiv:astro-ph/0109001.

[60] A. Cooray and R. Sheth, Phys. Rept. 372, 1 (Dec. 2002), arXiv:astro-ph/0206508.

[61] D. Schlegel, M. White, and D. Eisenstein, in astro2010: The Astronomy and Astrophysics Decadal Survey (2009), vol. 2010 of ArXiv Astrophysics e-prints, pp. 314-+, 0902.4680.

[62] D. J. Eisenstein, D. H. Weinberg, E. Agol, H. Aihara, C. Allende Prieto, S. F. Anderson, J. A. Arns, É. Aubourg, S. Bailey, E. Balbinot, et al., Astron. J. 142, 72, 72 (Sep. 2011), 1101.1529.

[63] M. White, M. Blanton, A. Bolton, D. Schlegel, J. Tinker, A. Berlind, L. da Costa, E. Kazin, Y.-T. Lin, M. Maia, et al., Astrophys. J. 728, 126, 126 (Feb. 2011), 1010.4915.

[64] Z. Zheng, A. A. Berlind, D. H. Weinberg, A. J. Benson, C. M. Baugh, S. Cole, R. Davé, C. S. Frenk, N. Katz, and C. G. Lacey, Astrophys. J. 633, 791 (Nov. 2005), arXiv:astro-ph/0408564.

[65] B. A. Reid, L. Samushia, M. White, W. J. Percival, M. Manera, N. Padmanabhan, A. J. Ross, A. G. Sánchez, S. Bailey, D. Bizyaev, et al., ArXiv e-prints (Mar. 2012), 1203.6641.

[66] R. Takahashi, N. Yoshida, T. Matsubara, N. Sugiyama, I. Kayo, T. Nishimichi, A. Shirata, A. Taruya, S. Saito, K. Yahata, et al., Mon. Not. Roy. Astron. Soc. 389, 1675 (Oct. 2008), 0802.1808.

[67] P. McDonald and U. Seljak, JCAP 10, 7 (Oct. 2009), 0810.0323.

[68] M. White, Y.-S. Song, and W. J. Percival, Mon. Not. Roy. Astron. Soc. 397, 1348 (Aug. 2009), 0810.1518 . 
[69] G. M. Bernstein and Y.-C. Cai, Mon. Not. Roy. Astron. Soc. 416, 3009 (Oct. 2011), 1104.3862.

[70] C. Park, Mon. Not. Roy. Astron. Soc. 319, 573 (Dec. 2000), arXiv:astro-ph/0012066.

[71] C.-G. Park and C. Park, Astrophys. J. 637, 1 (Jan. 2006), arXiv:astro-ph/0509740.

[72] R. E. Smith, R. Scoccimarro, and R. K. Sheth, Phys. Rev. D 75(6), 063512, 063512 (Mar. 2007), arXiv:astro-ph/0609547. 